A Simple and Fast Approach for Solving Problems on Planar Graphs

Fomin, F.V. and Thilikos, D.M.

Research Report LSI-03-54-R

Departament de Llenguatges i Sistemes Informàtics

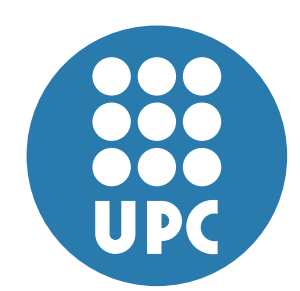

UNIVERSITAT POLITÈCNICA DE CATALUNYA 


\title{
A Simple and Fast Approach for Solving Problems on Planar Graphs ${ }^{* \dagger}$
}

\author{
Fedor V. Fomin Dimitrios M. Thilikos $^{\S}$
}

\begin{abstract}
It is well known that the celebrated Lipton-Tarjan planar separation theorem, in a combination with a divide-and-conquer strategy leads to many complexity results for planar graph problems. For example, by using this approach, many planar graph problems can be solved in time $2^{O(\sqrt{n})}$, where $n$ is the number of vertices. However, the constants hidden in big-Oh, usually are too large to claim the algorithms to be practical even on graphs of moderate size. Here we introduce a new algorithm design paradigm for solving problems on planar graphs. The paradigm is so simple that it can be explained in any textbook on graph algorithms: Compute tree or branch decomposition of a planar graph and do dynamic programming. Surprisingly such a simple approach provides faster algorithms for many problems. For example, InDEPENDENT SET on planar graphs can be solved in time $O\left(2^{3.182 \sqrt{n}} n+n^{4}\right)$ and Dominating SET in time $O\left(2^{5.043 \sqrt{n}} n+n^{4}\right)$. In addition, significantly broader class of problems can be attacked by this method. Thus with our approach, LONGEST CYCLE on planar graphs is solved in time $O\left(2^{2.29 \sqrt{n}(\ln n+0.94)} n^{5 / 4}+n^{4}\right)$ and BISECTION is solved in time $O\left(2^{3.182 \sqrt{n}} n+n^{4}\right)$. The proof of these results is based on complicated combinatorial arguments that make strong use of results derived by the Graph Minors Theory. In particular we prove that branch-width of a planar graph is at most $2.122 \sqrt{n}$. In addition we observe how a similar approach can be used for solving different fixed parameter problems on planar graphs. We prove that our method provides the best so far exponential speed-up for fundamental problems on planar graphs like VERTEX Cover, (Weighted) Dominating Set, and many others.
\end{abstract}

Keywords. Tree-width, Branch-width, Planar Graphs, Separation theorems, Fixed Parameter Algorithms, Independent Set, Vertex Cover, Dominating Set, Longest Cycle.

\footnotetext{
*This work was partially supported by the IST Program of the EU under contract number IST-199914186 (ALCOM-FT). The last author was supported by EC contract IST-1999-14186: Project ALCOM-FT (Algorithms and Complexity) - Future Technologies and by the Spanish CICYT project TIC-2002-04498C05-03 (TRACER)

${ }^{\dagger}$ A previous version of this paper (containing mainly the graph-theoretic results) appeared as Technical Report No. 240, Department of Informatics of Bergen University, Bergen, Norway, 2003 and as Technical Report LSI-02-56-R. Departament de Llenguatges i Sistemes Informàtics, Universitat Politècnica de Catalunya, Barcelona, Spain, 2002.

${ }^{\ddagger}$ Department of Informatics, University of Bergen, N-5020 Bergen, Norway, fomin@ii.uib.no

${ }^{\S}$ Departament de Llenguatges i Sistemes Informàtics, Universitat Politècnica de Catalunya, Campus Nord - Mòdul C5, c/Jordi Girona Salgado 1-3, E-08034, Barcelona, Spain, sedthilk@1si.upc.es
} 


\section{Introduction}

The design of (exponential) algorithms that are significantly faster than exhaustive search is one of the basic approaches of coping with NP-hardness [19]. Nice examples of fast exponential algorithms are Eppstein's graph coloring algorithm [18] and the algorithm for 3-SAT [12]. For a good overview of the field see the recent survey written by Gerhard Woeginger [35].

It is well known that by making use of the well-known approach of Lipton \& Tarjan [27] based on the celebrated planar separator theorem [26] one can obtain algorithms with time complexity $c^{O(\sqrt{n})}$ for many problems on planar graphs. However, the constants "hidden" in $O(\sqrt{n})$ can be crucial for practical implementations. During the last few years a lot of work has been done to compute and to improve the "hidden" constants [3, 4]. In this paper we observe a general approach for obtaining sub-exponential time exact algorithms for many problems on planar graphs. Our approach is based on dynamic programming for graphs of bounded branch-width (tree-width). Combining our upper bound for branchwidth of planar graphs with this simple approach one can obtain exponential speed-up for many known algorithms for many different planar graph problems. IndEPEndent Set, Dominating Set, SAT, MiN-Bisection, Longest Cycle (Path) on planar graphs are just a few examples of such problems.

Another field for implementation of our graph theoretical bounds is in the designing of parameterized algorithms. The last ten years were the evidence of rapid development of a new branch of computational complexity: Parameterized Complexity. (See the book of Downey \& Fellows [17].) Roughly speaking, a parameterized problem with parameter $k$ is fixed parameter tractable if it admits a solving algorithm with running time $f(k)|I|^{\beta}$. (Here $f$ is a function depending only on $k,|I|$ is the length of the non parameterized part of the input and $\beta$ is a constant.) Typically, $f(k)=c^{k}$ is an exponential function for some constant $k$. However, it appears, that for a large variety of planar graph problems algorithms with growth of the form $f(k)=c^{\sqrt{k}}$ are possible. During the last two years much attention was paid to the construction of algorithms with running time $c^{\sqrt{k}}$ for different problems on planar graphs. The first paper on the subject was the paper by Alber et al. [1] describing an algorithm with running time $O\left(4^{6 \sqrt{34 k}} n\right.$ ) (which is approximately $O\left(2^{70 \sqrt{k}} n\right)$ ) for the Planar Dominating Set problem. Different fixed parameter algorithms for solving problems on planar and related graphs are discussed in [4, 25]. We observe that our technique can serve also as a simple unified approach for solving many parameterized problems on planar graphs in subexponential time. Again, our approach is based on combinatorial bounds on planar branch-width and tree-width and provides a better running time for such basic parameterized problem like VerTex Cover, Dominating SET and many others.

The crucial part of our paper is devoted to the proof that such a simple approach guarantees better time bounds and here we use complicated combinatorial arguments 
coming from Robertson-Seymour's Graph Minor Theory. More precisely, our proof is based on a new upper bound to the branch-width and the tree-width of planar graphs. Both these parameters where introduced (and served) as basic tools by Robertson and Seymour in their Graph Minors series of papers. Tree-width and branch-width are related parameters (See Theorem 2.1) and can be considered as measures of the "global connectivity" of a graph. Moreover, they appear to be of a major importance in algorithmic design as many NP-hard problems admit polynomial or even linear time solutions when their inputs are restricted to graphs of bounded tree-width or branch-width. This motivated the search for graphs where these parameters are relatively small. In this direction, Alon, Seymour $\&$ Thomas proved in [6] that given a minor closed graph class $\mathcal{G}$, any $n$-vertex graph $G$ in $\mathcal{G}$ has tree-width/branch-width $O(\sqrt{n})$. As a consequence of this, any $n$-vertex planar graph $G$ has tree-width/branch-width $\leq 14.697 \sqrt{n}$.

We show that every $n$-vertex planar graph $G$ has branch-width $\leq 2.122 \sqrt{n}$ and treewidth $\leq 3.182 \sqrt{n}$. To our knowledge, this is the best known upper bound for the value of these parameters on planar graphs. To obtain the new upper bounds we use deep "dual" and "min-max" theorems from Graph Minors series papers of Robertson \& Seymour.

\subsection{Previous results and our contribution}

Computation of constants $\alpha_{t}$ and $\alpha_{b}$ such that for every planar graph on $n$ vertices $\operatorname{tw}(G) \leq$ $\alpha_{t} \sqrt{n}+O(1)$ and $\mathbf{b w}(G) \leq \alpha_{b} \sqrt{n}+O(1)$ is of a great theoretical importance. In [6] Alon, Seymour \& Thomas proved that any $K_{r}$-minor free graph on $n$ vertices has treewidth $\leq r^{1.5} \sqrt{n}$. (Here $K_{r}$ is complete graph on $r$ vertices.) Since no planar graph contains $K_{5}$ as a minor, we have that $\alpha_{t}(G) \leq 6^{1.5} \leq 14.697$.

Before we proceed, let us remind the notion of a minor. Given an edge $e=\{x, y\}$ of a graph $G$, the graph $G / e$ is obtained from $G$ by contracting the edge $e$; that is, to get $G / e$ we identify the vertices $x$ and $y$ and remove all loops and duplicate edges. A graph $H$ obtained by a sequence of edge-contractions is said to be a contraction of $G$. $H$ is a minor of $G$ if $H$ is the subgraph of a some contraction of $G$.

The following is a combination of statements (4.3) in [28] and (6.3) in [30].

Theorem 1.1 ([30]). Let $k \geq 1$ be an integer. Every planar graph with no $(k \times k)$-grid as a minor has branch-width $\leq 4 k-3$.

Because a graph on $n$ vertices does not contain a $((\lceil\sqrt{n}\rceil+1) \times(\lceil\sqrt{n}+1))$-grid as a minor, we have that $\alpha_{b}(G) \leq 4$. Robertson, Seymour, and Thomas showed (unpublished result announced by Thomas [34]) that any planar graph without a $(k \times k)$-grid as a minor has tree-width $\leq 5 k-1$ implying $\alpha_{t} \leq 5$.

To design the algorithms of this paper, we will reduce the constant $\alpha_{b}$ to 2.122 (for the case of branch-width) and $\alpha_{t}$ to 3.182 (for the case of tree-width). 
Lipton \& Tarjan [27] were first to observe the existence of time $2^{O(\sqrt{n})} n^{O(1)}$ algorithms for several problems on planar graphs. However the constants hidden in big-Oh of the exponent make these algorithms unpractical. Later, a lot of work was done on computing and reducing these constants. The best known so far results can be found in [4], where generalizations and complicated improvement of Lipton-Tarjan (together with kernel reduction techniques) are used to obtain subexponential parameterized algorithms.

Thus, for example, the approach suggested in [4] provides an $O\left(2^{9.07 \sqrt{n}} n \ln n\right)$ algorithm for Independent Set and an $O\left(2^{18.61 \sqrt{n}} n \ln n\right)$ algorithm for Dominating Set.

Here we suggest a unified approach based on branch decompositions (see Section 2 for the definitions). Our algorithm is simple and is performed in two steps: First we compute the branch decomposition of a planar graph and then do dynamic programming on graphs of bounded branch-width. Optimal branch decomposition of a planar graph can be constructed in polynomial time by using the algorithm due to Seymour \& Thomas (Sections 7 and 9 in [32]). (See also the results of Hicks [23] on implementations of Seymour \& Thomas algorithm.) For graphs with $n$ vertices this algorithm can be implemented in $O\left(n^{4}\right)$ steps. And what is important for practical applications, there is no large hidden constants in the running time of this algorithm. As for the second stage, well known dynamic programming algorithms on tree decompositions can be easily translated to branch decompositions. Using upper bounds for branch-width we prove that our approach provides more efficient solutions for many well known problems on planar graphs.

The following table summarize some known and new results on some problems on planar graphs (for more problems see Section 3). (See [33] for the definitions of PERFECT Code, $H$-Coloring, and $H$-COvering problems and Appendix for the definitions of other problems.)

\begin{tabular}{|c|c|c|}
\hline & Known results & New results \\
\hline Planar Independent Set & $O\left(2^{9.07 \sqrt{n}} n \ln n\right)[4]$ & $O\left(2^{3.182 \sqrt{n}} n+n^{4}\right)$ \\
\hline Planar Dominating Set & $O\left(2^{18.61 \sqrt{n}} n \ln n\right)[4]$ & $O\left(2^{5.043 \sqrt{n}} n+n^{4}\right)$ \\
\hline PLANAR $(k, r)$-CENTER & & $O\left((2 r+1)^{3.182 \sqrt{n}} n+n^{4}\right)$ \\
\hline Planar Longest Cycle & & $O\left(2^{2.29 \sqrt{n}(\ln n+0.94)} n^{5 / 4}+n^{4}\right)$ \\
\hline Planar Longest Path & & $O\left(2^{2.29 \sqrt{n}(\ln n+0.94)} n^{5 / 4}+n^{4}\right)$ \\
\hline Planar Bisection & & $O\left(2^{3.182 \sqrt{n}} n+n^{4}\right)$ \\
\hline Planar Weighted Dominating Set & & $O\left(2^{6.37 \sqrt{n}} n+n^{4}\right)$ \\
\hline Planar Perfect Code & & $O\left(2^{6.37 \sqrt{n}} n+n^{4}\right)$ \\
\hline Planar Total Dominating Set & & $O\left(2^{7.4 \sqrt{n}} n+n^{4}\right)$ \\
\hline PLANAR $H$-COLORING & & $O\left(2^{\log h \cdot 2.12 \sqrt{n}} h n^{3 / 2}+n^{4}\right)$ \\
\hline Planar Kernel & & $O\left(2^{3.37 \sqrt{n}} n^{2}+n^{4}\right)$ \\
\hline PLANAR $H$-COVERING & & $O\left(2^{9.55 \sqrt{n} h} n+n^{4}\right)$ \\
\hline
\end{tabular}

Similar approach works well also for parameterized problems. The next table summarize results on the most fundamental fixed parameter problems on planar graphs. (See [3] 
for an overview of the results on this subject.) We include the result from [20] because it is based on the main combinatorial result of this paper and is obtained by similar approach.

\begin{tabular}{|l|c|c|}
\hline & Known results & New results \\
\hline Planar $k$-Vertex Cover & $O\left(2^{4 \sqrt{3 k}} n\right)[3]$ & $O\left(2^{4.5 \sqrt{k}} k+k^{4}+k n\right)$ \\
\hline Planar $k$-Dominating Set & $O\left(2^{27 \sqrt{k}} n\right)[25]$ & $O\left(2^{15.13 \sqrt{k}} k+k^{4}+n^{3}\right)[20]$ \\
\hline Planar $k$-IndePendent Set & $O\left(2^{4 \sqrt{6 k}} n\right)[3]$ & $O\left(k^{4}+2^{4 \sqrt{4.5 k}} k+n\right)$ \\
\hline
\end{tabular}

Thus our approach provides exponential speedup for the main basic parameterized problems. Our method is quite universal and can be implemented to obtain an exponential speed-up for many known algorithms for different problems with fixed parameters. Mention just a few parameterized versions of the following problems: INDEPENDENT Dominating Set, Perfect Dominating Set, Perfect Code, Weighted Dominating Set, Total Dominating Set, Edge Dominating Set, Face Cover, Vertex FeedBack Set, Minimum Maximal Matching, Clique Transversal Set, Disjoint CyCles, and Digraph Kernel. Another advantage of our results is that they apply not only on planar graphs but on different generalizations of planar graphs, e.g. $K_{3,3}$-minor-free or $K_{5}$-minor-free graphs.

\section{Definitions and preliminary results}

All graphs in this paper are undirected, loop-less and, unless otherwise mentioned, they may have multiple edges.

\subsection{Tree-width and branch-width}

A tree decomposition of a graph $G$ is a pair $\left(\left\{X_{i} \mid i \in V(T)\right\}, T\right)$, where $\left\{X_{i} \mid i \in V(T)\right\}$ is a collection of subsets of $V(G)$ and $T$ is a tree, such that

1. $\bigcup_{i \in V(T)} X_{i}=V(G)$,

2. for each edge $\{v, w\} \in E(G)$, there is an $i \in V(T)$ such that $v, w \in X_{i}$, and

3. for each $v \in V(G)$ the set of nodes $\left\{i \mid v \in X_{i}\right\}$ forms a subtree of $\mathrm{T}$.

The width of a tree decomposition $\left(\left\{X_{i} \mid i \in V(T)\right\}, T\right)$ equals $\max _{i \in V(T)}\left(\left|X_{i}\right|-1\right)$. The tree-width of a graph $G, \operatorname{tw}(G)$, is the minimum width over all tree decompositions of $G$.

A branch decomposition of a graph (or a hyper-graph) $G$ is a pair $(T, \tau)$, where $T$ is a tree with vertices of degree 1 or 3 and $\tau$ is a bijection from the set of leaves of $T$ to $E(G)$. The order of an edge $e$ in $T$ is the number of vertices $v \in V(G)$ such that there are leaves $t_{1}, t_{2}$ in $T$ in different components of $T(V(T), E(T)-e)$ with $\tau\left(t_{1}\right)$ and $\tau\left(t_{2}\right)$ both containing $v$ as an endpoint. 
The width of $(T, \tau)$ is the maximum order over all edges of $T$, and the branch-width of $G$, $\mathbf{b w}(G)$, is the minimum width over all branch decompositions of $G$. (In case where $|E(G)| \leq 1$, we define the branch-width to be 0 ; if $|E(G)|=0$, then $G$ has no branch decomposition; if $|E(G)|=1$, then $G$ has a branch decomposition consisting of a tree with one vertex - the width of this branch decomposition is considered to be 0 ).

It is known from [28] that if $H$ is a minor of $G$ then $\mathbf{b w}(H) \leq \mathbf{b w}(G)$. The following result is due to Robertson \& Seymour [(5.1) in [28]].

Theorem 2.1 ([28]). For any connected graph $G$ where $|E(G)| \geq 3$, bw $(G) \leq \mathbf{t w}(G)+$ $1 \leq \frac{3}{2} \mathbf{b w}(G)$.

From Theorem 2.1, any upper bound on tree-width implies an upper bound on branchwidth and vice versa.

\subsection{Planar graphs, slopes and majorities}

In this paper we use the expression $\Sigma$-plane graph for any planar graph drawn in the sphere $\Sigma$. To simplify notations we do not distinguish between a vertex of a $\Sigma$-plane graph and the point of $\Sigma$ used in the drawing to represent the vertex or between an edge and the open line segment representing it. We also consider $G$ as the union of the points corresponding to its vertices and edges. That way, a subgraph $H$ of $G$ can be seen as a graph $H$ where $H \subseteq G$. We call by region of $G$ any connected component of $\Sigma-E(G)-V(G)$. (Every region is an open set.) We use the notation $V(G), E(G)$, and $R(G)$ for the set of the vertices, edges and regions of $G$. A path of $G$ is any connected subgraph $P$ of $G$ with two vertices of degree 1 (we call them extremes) and all other vertices (we call them internal) of degree 2. A sub-path of a path $P$ is any path $P^{\prime} \subseteq P$. A cycle of $G$ is any connected subgraph $C$ of $G$ with all the vertices of degree 2. The length $|C|(|P|)$ of a cycle $C$ (path $|P|)$ is the number of its edges.

If $\Delta \subseteq \Sigma$, then $\bar{\Delta}$ denotes the closure of $\Delta$, and the boundary of $\Delta$ is $\operatorname{bd}(\Delta)=$ $\bar{\Delta} \cap \overline{\Sigma-\Delta}$. An edge $e$ (a vertex $v$ ) is incident with a region $r$ if $e \subseteq \mathbf{b d}(r)(v \subseteq \mathbf{b d}(r))$.

We call a $\Sigma$-plane graph $G$ triangulated if all of its regions are triangles, i.e. for every region $r, \mathbf{b d}(r)$ is a cycle of three edges and three vertices. Given a region $r$ of a triangulated graph $G$ we call the cycle bd $(r)$ triangle of $G$. A triangulation $H$ of a $\Sigma$-plane graph $G$ is any triangulated $\Sigma$-plane graph $H$ where $G \subseteq H$. Notice that any $\Sigma$-plane graph with all regions of size $\geq 3$ has a triangulation. A triangle of a triangulated $\Sigma$-plane graph $G$ is a regional triangle if it bounds a region of $G$.

Let $G$ be a $\Sigma$-plane graph. A subset of $\Sigma$ meeting the drawing only in vertices of $G$ is called $G$-normal. A subset of $\Sigma$ homeomorphic to the closed interval $[0,1]$ is called $I$-arc. If the extreme points of a $G$-normal $I$-arc $L$ are both vertices of $G$ then we call it line of $G$. If a simple closed curve $F \subseteq \Sigma$ is $G$-normal then we call it noose.

The length of a line is the number of its vertices minus 1 and the length of a noose is the number of its vertices. We denote by $|N|(|L|)$ the length of a noose $N$ (line $L$ ). 
$\Delta \subseteq \Sigma$ is an open disc if it is homeomorphic to $\left\{(x, y): x^{2}+y^{2}<1\right\}$. We say that a disc $D$ is bounded by a noose $N$ if $N=\mathbf{b d}(D)$. From the theorem of Jordan, any noose $N$ bounds exactly two closed discs $\Delta_{1}, \Delta_{2}$ in $\Sigma$ where $\Delta_{1} \cap \Delta_{2}=N$. We call $\Theta$-structure $S=\left(L_{1}, L_{2}, L_{3}\right)$ of $G$ the union of three mutually touching lines. If for $i, j, 1 \leq i<j \leq 3$ the noose $L_{i} \cup L_{j}$ has size $\leq k$ then we say that $S$ is a $\Theta$-structure of length $\leq k$. We call a $\Theta$-structure non-trivial if at least two of its lines have length $\geq 2$. We call the 6 closed discs bounded by the nooses $L_{i} \cup L_{j}, 1 \leq i<j \leq 3$ closed discs bounded by $S$.

The radial graph of a $\Sigma$-plane graph $G$ is the bipartite $\Sigma$-plane graph $R_{G}$ obtained by selecting a point in every region $r$ of $G$ and connecting it to every vertex of $G$ incident to that region. We call the vertices of $R_{G}$ that are not vertices of $G$ radial vertices. For an example of a graph $G$ drawn along with its radial, see Fig. 1 in the Appendix.

Slopes and majorities are important tools for improving upper bounds.

Slopes (Robertson \& Seymour [29]). Let $G$ be a $\Sigma$-plane graph and let $k \geq 1$ be an integer. A slope in $G$ of order $k / 2$ is a function ins which assigns to every cycle $C$ of $G$ of length $<k$ one of the two closed $\operatorname{discs} \operatorname{ins}(C) \subseteq \Sigma$ bounded by $C$ such that

[S1] If $C, C^{\prime}$ are cycles of length $<k$ and $C \subseteq \operatorname{ins}\left(C^{\prime}\right)$ then $\operatorname{ins}(C) \subseteq \operatorname{ins}\left(C^{\prime}\right)$.

[S2] If $P_{1}, P_{2}, P_{3}$ are three paths of $G$ joining the same pair $u, v$ of distinct vertices but otherwise disjoint, and the three cycles $P_{1} \cup P_{2}, P_{1} \cup P_{3}, P_{2} \cup P_{3}$ all have length $<k$ then

$$
\operatorname{ins}\left(P_{1} \cup P_{2}\right) \cup \operatorname{ins}\left(P_{1} \cup P_{3}\right) \cup \operatorname{ins}\left(P_{2} \cup P_{3}\right) \neq \Sigma \text {. }
$$

A slope is uniform if for every region $r \in R(G)$ there is a cycle $C$ of $G$ of length $<k$ such that $r \subseteq \operatorname{ins}(C)$.

We need the following deep result proved in the Graph Minors papers by Robertson \& Seymour. This result follows from Theorems (6.1) and (6.5) in [29] and Theorem (4.3) in [28]. (See also Theorems (6.2) and (7.1) in [32].)

Theorem 2.2 ([29]). Let $G$ be a connected and loopless $\Sigma$-plane graph where $|E(G)| \geq 2$ and let $k \geq 1$ be an integer. The radial drawing $R_{G}$ has a uniform slope of order $\geq k$ if and only if $G$ has branch-width $\geq k$.

Majorities (Alon, Seymour \& Thomas [7]). Let $G$ be a $\Sigma$-plane graph and let $k \geq 0$ be an integer. A majority of order $k$ is a function big that assigns to every noose $N$ of length $\leq k$ a closed $\operatorname{disc} \operatorname{big}(N) \subseteq \Sigma$ bounded by $N$ such that

[M1] If $P_{1}, P_{2}, P_{3}$ is a $\Theta$-structure of $G$ with length $\leq k$ and $P_{3} \subseteq \operatorname{big}\left(P_{1} \cup P_{2}\right)$, then $\operatorname{big}\left(P_{1} \cup P_{3}\right) \subseteq \operatorname{big}\left(P_{1} \cup P_{2}\right)$ or $\operatorname{big}\left(P_{2} \cup P_{3}\right) \subseteq \operatorname{big}\left(P_{1} \cup P_{2}\right)$.

[M2] If $N$ is a noose of length $\leq \min (2, k)$ then either $\operatorname{big}(N)-N$ contains a vertex or $\operatorname{big}(N)$ includes at least two edges of $G$. 
The following result gives an upper bound on the order of a majority (statement (3.7) of [7]). This is a basic ingredient of our bound for the branch-width of planar graphs.

Theorem 2.3 ([7]). Any majority of a $\Sigma$-plane graph $G$ has order $\sqrt{4.5 \cdot|V(G)|}-1$.

Our bounds on branch-width and tree-width follows from the following theorem that is the main combinatorial result of the paper.

Theorem 2.4. Let $G,|V(G)| \geq 5$, be a triangulated $\Sigma$-plane graph without multiple edges, drawn in $\Sigma$ along with its radial graph and let $k \geq 2$ be an integer. If there exists a uniform slope of order $k+1$ in $R_{G}$ then $G$ contains a majority of order $k$.

The proof of Theorem 2.4 is rather long and technical. Due to space restrictions we sketch here the main ideas of the proof. (The detailed proof has been moved to the Appendix.)

\subsection{The ideas of the proof of Theorem 2.4.}

We want to correspond nooses of $G$ to cycles of $R_{G}$ and try to translate the slope axioms to majority axioms. Corresponding nooses to cycles is not direct as not every noose is a cycle of the radial graph. To overcome this problem we need to work with "classes" of similar structures.

Let $G$ be a $\Sigma$-plane graph without loops or multiple edges and let $S \subseteq \Sigma$ be an $I$ arc (simple closed curve) in $\Sigma$. We use the notation $\kappa_{G}(S)=\left(v_{1}, \ldots, v_{|S \cap V(G)|}\right)$ for the ordering (cyclic ordering) of the vertex set $S \cap V(G)$ that represents the way the vertices of $G$ are met by $S$. Notice that $\kappa$ can be applied to both cycles and nooses but also to paths and lines. Especially for cycles and paths of graphs without multiple edges, we can directly represent them with the output of the function $\kappa$ (we will use the same notation for a cycle/path and the (cyclic) ordering of the vertices that it meets).

Let $S$ be one of the following structures in $G$ : a noose, a line, or a $\Theta$-structure. A variation of $S$ is the operation that transforms $S$ to a structure $S^{\prime}$ of the same type in a way that $\operatorname{dif}\left(S, S^{\prime}\right):=\overline{\left(S \cup S^{\prime}\right)-\left(S \cap S^{\prime}\right)}$ is a noose of size 2 where one of the closed discs $D$ it bounds has the following two properties:

1. $D-\mathbf{b d}(D)$ contains no vertices of $G$,

2. $D$ contains at most one edge of $G$.

If two structures $S_{1}$ and $S_{2}$ are variations each of the other, we denote it as $S_{1} \sim S_{2}$. If a structure $S^{\prime}$ is the result of a finite number of consecutive variations with $S$ as starting point, we call $S^{\prime}$ vibration of $S$ and we denote this fact as $S \sim^{*} S^{\prime}$. (See Fig. 1 in the Appendix.) Notice that if $S \sim^{*} S^{\prime}$ then $V(G) \cap S=V(G) \cap S^{\prime}$ and $S, S^{\prime}$ have the same length. 
The importance of vibrations is that in a triangulated $\Sigma$-plane graph without multiple edges every noose is a vibration of a cycle of the radial graph. This fact is intuitively clear but needs a technical proof. (We move this proof to the Appendix, Lemma B.7.)

Let ins be a uniform slope of order $k+1$ in $R_{G}$. To construct a majority we need to define the function big. Every noose $N$ in $\Sigma$ of size $\leq k$ is a vibration of a cycle $C$ in $R_{G}$ and the length of $C$ is $\leq 2 k$. Cycle $C$ is also a noose in $\Sigma$ and because $C$ and $N$ are vibrations of each other, they "separate" the same vertex sets in $G$. In other words, if ins $(C), \overline{\Sigma-\operatorname{ins}(C)}$ are closed discs bounded by $C$ then for one of the closed discs $D$ bounded by $N$, we have that $D \cap V(G)=\overline{\Sigma-\operatorname{ins}(C)} \cap V(G)$. We define $\operatorname{big}(N)=D$.

The proof of the fact that the function big defined via ins satisfies majority axioms is quite technical. It uses some results about vibrations of $\Theta$-structures. (These results are moved to the Appendix, Section B.4.) and requires a series of auxiliary results assuring that the basic topological properties involved in the majority axioms are invariants under vibrations. (Section B.6 of the Appendix.)

Theorem 2.4 implies our main combinatorial result.

Theorem 2.5. For any planar graph $G$, bw $(G) \leq \sqrt{4.5|V(G)|} \leq 2.122 \sqrt{|V(G)|}$.

Proof. We assume that $G$ has no multiple edges (notice that the duplication of an edge does not increase the branch-width of a graph with branch-width $\geq 2$ ). It is easy to see that $G$ has a triangulation $H$ without multiple edges. It is enough to prove the bound of the theorem for $H$. By Theorem 2.3, $H$ does not have any majority of order $\geq(3 / \sqrt{2}) \sqrt{|V(G)|}$. By Theorem 2.4, $R_{H}$ has no slope of order $\geq(3 / \sqrt{2}) \sqrt{|V(G)|}+1$. The result now follows from Theorem 2.2.

Since $9 /(2 \sqrt{2})<3.182$, Theorems 2.1 and 2.5 imply the following:

Theorem 2.6. For any planar graph $G$, tw $(G) \leq 3.182 \sqrt{|V(G)|}$.

In the next section examine the algorithmic consequences of our combinatorial bounds.

\section{Algorithmic consequences}

In this section we discuss some applications of our results for different problems on planar graphs.

\subsection{Exact algorithms}

The following simple theorem is the source for obtaining subexponential algorithms for many graph problems.

Theorem 3.1. Let $\Pi$ be an optimization problem that is solvable on graphs of branch-width $\leq \ell$ in time $f(\ell) g(n)$. Then on planar graphs problem $\Pi$ is solvable in time $O(f(2.122 \sqrt{n}) g(n)+$ $\left.n^{4}\right)$ 
Proof. First we compute an optimal branch decomposition of planar graph. To compute an optimal branch decomposition of a planar graph one can use the algorithm due to Seymour \& Thomas (Sections 7 and 9 in [32]). (See also the results of Hicks [23] on implementations of Seymour \& Thomas algorithm.) This algorithm can be implemented in $O\left(n^{4}\right)$ steps. Then Theorem 2.5 implies the proof.

Corollary 3.2. Let $\Pi$ be an optimization problem that is solvable on graphs of branchwidth/tree-width $\leq \ell$ in time $2^{o\left(\ell^{2}\right)}$ poly $(n, \ell)$. Then on planar graphs problem $\Pi$ is solvable in subexponential time (in $2^{o(n)}$ steps).

In spite of its simplicity, Theorem 3.1 provides a general framework for obtaining subexponential algorithms for a broad range of problems. And the only thing one needs to know to estimate the running time of the algorithm is how fast a problem can be solved on graphs of bounded branch-width/tree-width ${ }^{1}$. But really surprising is that such a trivial approach provides better time estimation than many, complicated to analyze, algorithms based on separator theorems.

Let us give just few examples. It is well known that on graphs of tree-width $\ell$ INDEPENDENT SET can be solved in time $O\left(2^{\ell} n\right)$ and hence on graphs of branch-width $\leq \ell$ it can be solved in time $O\left(2^{(3 / 2) \ell} n\right)$. Thus by Theorem 3.1 we obtain that InDEPENDENT SET on planar graphs is solvable in $O\left(2^{3.182 \sqrt{n}} n+n^{4}\right)$. Dominating SET on graphs of branch-width $\leq \ell$ is solvable is time $O\left(2^{3 \log _{4} 3 \cdot \ell} m\right)$ [13]. Thus on planar graphs, DomINATING SET is solvable in $O\left(2^{5.043 \sqrt{n}} n+n^{4}\right)$. Similar arguments, based on the algorithms in [5], work for for the planar versions of different variations of the Dominating SET problem like Independent Dominating Set, Perfect Dominating Set, Perfect Code, Weighted Dominating Set, Red Blue Dominating Set where the time is $O\left(2^{6.37 \sqrt{n}} n+n^{4}\right)$, and for Total Dominating Set and Total Perfect Dominating SET where the time is $O\left(2^{7 \cdot 4 \sqrt{n}} n+n^{4}\right)$.

LONGEST CYCLE and LONGEST PATH problems on graphs of tree-width $\ell$ are solved in $O\left(\ell ! 2^{\ell} n\right)$ time [8] implying an $O\left(2^{2.29 \sqrt{n}(\ln n+0.94)} n^{5 / 4}+n^{4}\right)$ algorithm on planar graphs ${ }^{2}$. MIN-BISECTION is solvable in $O\left(2^{\ell} n\right)$ [24] on graphs of tree-width $\ell$ and the planar version of the problem is solvable in $O\left(2^{3.182 \sqrt{n}} n+n^{4}\right)$. In [21], Gutin et al. gave a time $O\left(3^{\ell} k n\right)$ algorithm for finding a kernel of size $k$ in a digraph whose underlying graph has treewidth at most $\ell$. This implies that Kernel is solvable in $O\left(2^{3.37 \sqrt{n}} n^{2}+n^{4}\right)$. The $H$-COLORING problem is solvable in $O\left(h^{\ell+1} \ell n\right)$ on graphs of tree-width $\ell$ [15], therefore its planar version is solvable in time $O\left(2^{\log h \cdot 2 \cdot 12 \sqrt{n}} h n^{3 / 2}+n^{4}\right)$. H-Cover is solvable in time $O\left(n 2^{3 \ell h}\right)$ [33] on graphs of tree-width $\leq \ell$ and thus for planar graphs in time $O\left(2^{9.546 \sqrt{n} h} n+n^{4}\right)$. Finally,

\footnotetext{
${ }^{1}$ Let us remark that any algorithm solving a problem on graphs of tree-width $\leq \ell$ in time $f(\ell) g(n)$ can be easy translated to the algorithm for graphs of branch-width $\leq \ell$ with running time $O(f(3 / 2 \ell) g(n)+m)$ where $m$ is the number of edges of the input graph.

${ }^{2}$ The calculation of the exponent in this algorithm makes use of Stirling's formula.
} 
$(k, r)$-CENTER is solvable in time $O\left((2 r+1)^{\frac{3}{2} \cdot \ell} m\right)$ on graphs of branch-width $\leq \ell[13]$ providing an $O\left((2 r+1)^{3.182 \sqrt{n}} n+n^{4}\right)$ algorithm for the planar version of the problem.

More generally, almost every natural problem expressible in MSOL is solvable in time $O\left(c^{\ell} n^{O(1)}\right), O\left(\ell^{\ell} n^{O(1)}\right)$ or $O\left(\ell ! c^{\ell} n^{O(1)}\right)$, and by Corollary 3.2 is solvable in subexponential time on planar graphs. Examples of such problems where $c$ is a small constant are Vertex Feedback Set, Disjoint Cycles, Face Cover. Edge Dominating Set, Clique Transversal, and Maximal Maching (see [10, 14]). For all these problems Corollary 3.2 provides subexponential algorithms with small hidden constants.

Actually, one can further strengthen the conditions of Corollary 3.2 towards extending the framework where subexponential algorithms are possible. Indeed, it is enough to have a time $(\operatorname{poly}(\ell, n))^{o\left(\ell^{2}\right)}$ algorithm for the problem $\Pi$ for graphs of treewidth/branchwidth at most $\ell$. Notice that such problems are not necessarily expresible in MSOL. As an example we mention the problems of finding a non-preemptive multicoloring with minimum sum/makespan (see Appendix for the definitions). These problems can be solved in time $O\left(n \cdot(\ell p \log n)^{\ell+1}\right)$ for graphs with tree-width $\leq \ell$ (see [22]). Therefore, they can be solved in time $O\left(p n^{3 / 2} \log n \cdot 2^{1.15 \cdot \log p \log n \log \log n \sqrt{n}}+n^{4}\right)$ on planar graphs.

\subsection{Parameterized algorithms}

Similar ideas work for parameterized problems. Let $\mathcal{L}$ be a parameterized problem, i.e. $\mathcal{L}$ consists of pairs $(I, k)$ where $k$ is the parameter of the problem. Reduction to linear problem kernel is the replacement of problem inputs $(I, k)$ by a reduced problem with inputs $\left(I^{\prime}, k^{\prime}\right)$ (linear kernel) with constants $c_{1}, c_{2}$ such that

$$
k^{\prime} \leq c_{1} k,\left|I^{\prime}\right| \leq c_{2} k^{\prime} \text { and }(I, k) \in \mathcal{L} \Leftrightarrow\left(I^{\prime}, k^{\prime}\right) \in \mathcal{L} .
$$

(We refer to Downey \& Fellows [17] for discussions on fixed parameter tractability and the ways of constructing kernels.)

Theorem 3.3. Let $\mathcal{L}$ be a parameterized problem $(I, k)$ (here $I$ can be a graph, hypergraph or matroid) such that

- There is a linear problem kernel computable in time $T_{k e r n e l}(|I|, k)$ with constants $c_{1}$, $c_{2}$ and such that an optimal branch decomposition of the kernel is computable in time $T_{b w}\left(\left|I^{\prime}\right|\right)$.

- On graphs (hypergraphs, matroids) of branch-width $\leq \ell$ and ground set of size $n$ the problem $\mathcal{L}$ can be solved in $O\left(2^{c_{3} \ell} n\right)$, where $c_{3}$ is a constant.

- bw $\left(I^{\prime}\right) \leq c_{4} \sqrt{k}$, where $c_{4}$ is a constant. Then $\mathcal{L}$ can be solved in time $O\left(2^{c_{3} c_{4} \sqrt{k}} k+\right.$ $\left.T_{b w}\left(\left|I^{\prime}\right|\right)+T_{\text {kernel }}(|I|, k)\right)$.

Proof. The algorithm works as follows. First we compute a linear kernel in time $T_{\text {kernel }}(|I|, k)$. Then we construct a branch decomposition of the kernel in $T_{b w}\left(\left|I^{\prime}\right|\right)$ steps. The size of the kernel is at most $c_{1} c_{2} k=O(k)$. The branch-width of the kernel is at most $c_{4} \sqrt{k}$ and it takes $O\left(2^{c_{3} c_{4} \sqrt{k}} k+T_{b w}\left(\left|I^{\prime}\right|\right)+T_{\text {kernel }}(|I|, k)\right)$ to solve the problem. 
Let us give some examples, where Theorem 3.3 provides proven better bounds for different parameterized problems.

The Planar $k$-Vertex Cover problem is the task to compute, given a planar graph $G$ and a positive integer $k$, a vertex cover of size $k$ or to report that no such a set exists. A linear problem kernel of size $2 k$ (with constants $c_{1}=1$ and $c_{2}=2$ ) for the $k$-VERTEX Cover problem (not necessary planar) was obtained by Chen et al. [11]. The running time of the algorithm constructing a kernel of a graph on $n$ vertices is $O\left(k n+k^{3}\right)$. So in this case $T_{\text {kernel }}(|I|, k)=O\left(k n+k^{3}\right)$. It is well known that the VerTex Cover problem on graphs on $n$ vertices and with bounded tree-width $\leq \ell$ can be solved in $O\left(2^{\ell} n\right)$ time. The dynamic programming algorithm for the VERTEx Cover on graphs with bounded tree-width can be easy translated to the dynamic programming algorithm for graphs with bounded branch-width with running time $O\left(2^{3 / 2 \ell} \mathrm{m}\right)$, where $m$ is the number of edges in a graph, and we omit it here. For planar graphs $2^{3 / 2 \ell} m=O\left(2^{3 / 2 \ell} n\right)$, thus $c_{3} \leq 3 / 2$.

From the constructions used in the reduction algorithm of Chen et al. [11] it follows that if $G$ is a planar graph then the kernel graph is also planar. To compute an optimal branch decomposition of a planar graph one can use the algorithm due to Seymour \& Thomas [32]. This algorithm (applied to the kernel graph) can be implemented in $O\left(k^{4}\right)$ steps. The kernel graph $I^{\prime}$ has at most $2 k$ vertices. Then by Theorem $2.5, c_{4} \leq \sqrt{4.5} \sqrt{2}=$ 3. Thus by making use of Theorem 3.3, we conclude that Planar $k$-Vertex Cover can be solved in $O\left(k^{4}+2^{4.5 \sqrt{k}} k+k n\right)$.

A $k$-dominating set $D$ of a graph $G$ is a set of $k$ vertices such that every vertex outside $D$ is adjacent to a vertex of $D$. The Planar $k$-Dominating Set problem is the task to compute, given a planar graph $G$ and a positive integer $k$, a $k$-dominating set or to report that no such a set exists.

Alber, Fellows \& Niedermeier [2] show that the Planar Dominating Set problem admits a linear problem kernel. (The size of the kernel is 335k.) This reduction can be performed in $O\left(n^{3}\right)$ time. Dominating Set problem on graphs of branch-width $\leq \ell$ can be solved in $O\left(2^{3 \log _{4} 3 \cdot \ell} m\right)$ steps [20]. Thus $c_{3} \leq 3 \log _{4} 3$. It is proved in [20] that for every planar graph $G$ with dominating set $k$, the branch-width of $G$ is at most $3 \sqrt{4.5} \sqrt{k}$, i.e. $c_{4} \leq 3 \sqrt{4.5}$. Then by Theorem 3.3, Planar Dominating set can be solved in $O\left(2^{15.13 \sqrt{k}} k+n^{3}+k^{4}\right)$.

\subsection{Other problems and generalizations.}

Our ideas can be adapted to different problems by using the bounds and tree-width (branch-width) based algorithms in the same fashion as it is done in $[1,3,10,14]$. That way, our upper bound implies the construction of faster algorithms for a series of problems when their inputs are restricted to planar graphs. As a sample we mention parameterized versions of the following problems: Independent Dominating Set, Perfect Dominating Set, Perfect Code, Weighted Dominating Set, Total Dominat- 
ing Set, Edge Dominating Set, Face Cover, Vertex Feedback Set, Minimum Maximal Matching, Clique Transversal Set, Disjoint Cycles, and Digraph KeRnel (see $[1,3,10,14]$ for the exact definitions).

Finally let us note that our upper bound for treewidth holds not only on planar graphs but on different generalizations of planar graphs. This follows directly from the results of [14] and implies an exponential speed-up of all the aforementioned problems on certain classes of non-planar graphs such as $K_{3,3}$-minor-free or $K_{5}$-minor-free graphs.

\section{Discussion and open problems}

In this section we present three open problems emerging from our main combinatorial result and the methodology of our proof.

Improving the constant 2.122. According to Theorem 2.5, any planar graph on $n$ vertices has branch-width $\leq 2.122 \sqrt{n}$. The constant 2.122 follows from the constant of Theorem 2.3 proven by Alon, Seymour, and Thomas in [7]. Any improvement of the constant of Theorem 2.3 implies also an improvement of our bound.

Given a graph $G$, a function $w: V(G) \rightarrow \mathbb{R}$, and a set $S \subseteq V(G)$, we call $S(2 / 3)$ separator of $G$ if $V(G)-S$ can be partitioned into two sets $A_{1}, A_{2}$ where no edge of $E(G)$ has one endpoint in $A_{1}$ and the other in $A_{2}$ and such that $w\left(A_{i}\right) \leq \frac{2}{3} w(V(G))$. If we strengthen the definition of a (2/3)-separator by asking that $w\left(A_{i}\right)+\frac{1}{2} w(S) \leq \frac{2}{3} w(V(G))$, we define the notion of a strong (2/3)-separator of $G$. If $G$ is $\Sigma$-plane and there exist a noose $N$ bounding the open discs $D, D^{\prime}$ such that $D \cap V(G)=A_{1}, D^{\prime} \cap V(G)=A_{2}$, and $S=N \cap V(G)$ then we call $S$ (strong) cyclic (2/3)-separator of $G$.

In [7], Alon, Seymour and Thomas proved the following.

Theorem 4.1. Let $G$ be a $\Sigma$-plane graph with $n$ vertices, let $w: V(G) \rightarrow \mathbb{R}$ be a function, and let $k \geq 0$ be an integer. If every majority of $G$ has order $\leq k$ then $G$ has a strong $(2 / 3)$-separator of $G$ of size $\leq k$.

Theorems 4.1 and 2.3 were proved in [7] in order to imply the following.

Theorem 4.2. Let $G$ be a $\Sigma$-plane graph with $n$ vertices and let $w: V(G) \rightarrow \mathbb{R}$ be a function. Then $G$ has strong cyclic (2/3)-separator of size $\leq 2.122 \sqrt{n}$.

Curiously, any proof of Theorem 4.2 for a better constant $c$, implies the reduction of the constant of Theorem 2.5 from 2.122 to $\max \{2, c\}$. Indeed, this is correct because of Theorems 2.2 and 2.5 and the following interesting result (statement (3.9) of [7]).

Theorem 4.3. Let $G$ be a $\Sigma$-plane graph with $n$ vertices, let $w: V(G) \rightarrow \mathbb{R}$ be a function, and let $k$ be an integer where $k \geq 2 \sqrt{n}-1$. If $G$ contains a strong (2/3)-cyclic separator of size $\leq k$ then every majority of $G$ has order $\leq k$. 
In [16], Djidjev \& Venkatesan proved that every $\Sigma$-plane graph on $n$ vertices contains a cyclic $2 / 3$-separator of size $2 \sqrt{n}+O(1)$. It is an interesting challenge to strengthen this result so that it guarantees the existence of a strong cyclic $(2 / 3)$-separator, as required by Theorem 4.2. This would make it possible to reduce to 2 the constant 2.122 of our main result (and to improve the time bounds of our algorithms).

Creating slopes from majorities. We believe that the ideas of this paper can be useful for proving the following conjecture.

Conjecture. Any planar graph $G$ has a cyclic $(2 / 3)$-separator of size $\leq \mathbf{b w}(G)$.

Conjecture 4 can follow from Theorems 2.2 and 4.1 if the inverse of Theorem 2.4 holds for general graphs. In this direction, one should show that majorities can be "transformed" to slopes. As any cycle $C$ of $R_{G}$ is also a noose of $G$ we can directly define $\operatorname{ins}(C)=$ $\overline{\Sigma-\operatorname{big}(C)}$, following the idea in the proof of Theorem 2.4 (notice that in this direction the idea does not need the "vibration" machinery). Moreover it is possible to prove that the axiom [M2] for big implies the uniformity of ins and axiom [M1] for big implies axiom [S2] for ins. However, it is not easy to prove that axiom [S1] also holds for ins and this is the main obstacle for any proof of Conjecture 4 based on the possible "translation" of majorities to slopes.

Constructive upper bounds. While Theorem 2.5 gives an upper bound to the branch-width of any planar graph, it does not provide any way to construct the corresponding branch decomposition. The "non-constructiveness" of our proof emerges from the fact that it makes strong use of the results in [7], [28] and [30] that are not (at least directly) "translatable" to a polynomial time algorithm. However, the algorithmic results of [30] make it possible to construct, for any $n$-vertex planar graph, a branch decomposition of width $\leq 2.122 \sqrt{n}$ in time $O\left(n^{4}\right)$ and such a branch decomposition can be easily transformed to a tree decomposition of width $\leq 3.128 \sqrt{n}$ using the results of [29]. It is an open problem, whether Theorem 2.5 can admit a simple proof implying faster algorithms for the construction of the corresponding decompositions. Robin Thomas (in private communication) mentioned that by adapting the arguments from [7] one can obtain similar bounds on branch-width/tree-width. Perhaps this can bring us to faster algorithms.

\section{References}

[1] J. Alber, H. L. Bodlaender, H. Fernau, T. Kloks, and R. Niedermeier, Fixed parameter algorithms for dominating set and related problems on planar graphs, Algorithmica, 33 (2002), pp. 461-493.

[2] J. Alber, M. R. Fellows, and R. Niedermeier, Efficient data reduction for dominating set: A linear problem kernel for the planar case, in SWAT 2002, Springer, vol. 2368, Berlin, 2002, pp. 150-159. 
[3] J. Alber, H. Fernau, and R. Niedermeier, Parameterized complexity: Exponential speed-up for planar graph problems, in Electronic Colloquium on Computational Complexity (ECCC), Germany, 2001.

[4] J. Alber, H. Fernau, and R. Niedermeier, Graph separators: a parameterized view, J. of Computer and System Sciences, (2003), p. to appear.

[5] J. Alber And R. Niedermeier, Improved tree decomposition based algorithms for domination-like problems, in Latin American Theoretical Informatics-LATIN 2002 (Cancun, Mexico), Springer, Lecture Notes in Computer Science, vol. 2286, Berlin, 2002, pp. 613-627.

[6] N. Alon, P. Seymour, And R. Thomas, A separator theorem for nonplanar graphs, J. Amer. Math. Soc., 3 (1990), pp. 801-808.

[7] — Planar separators, SIAM J. Discrete Math., 7 (1994), pp. 184-193.

[8] H. L. Bodlaender, On linear time minor tests with depth-first search, J. Algorithms, 14 (1993), pp. 1-23.

[9] D. Bullock and C. Hendrickson, Roadway traffic control software, IEEE Transactions on Control Systems Technology, 2 (1994), pp. 255-264.

[10] M.-S. Chang, T. Kloks, And C.-M. Lee, Maximum clique transversals, in WG 2001, vol. 2204 of Lecture Notes in Comput. Sci., Springer, Berlin, 2001, pp. 32-43.

[11] J. Chen, I. A. Kanj, And W. Jia, Vertex cover: further observations and further improvements, J. Algorithms, 41 (2001), pp. 280-301.

[12] E. Dantsin, A. Goerdt, E. A. Hirsch, R. Kannan, J. Kleinberg, C. PaPadimitriou, P. Raghavan, And U. Schöning, A deterministic $(2-2 /(k+1))^{n}$ algorithm for $k$-SAT based on local search, Theoret. Comput. Sci., 289 (2002), pp. 6983.

[13] E. D. Demaine, F. V. Fomin, M. Hajiaghayi, and D. M. Thilikos, Fixedparameter algorithms for the $(k, r)$-center in planar graphs and map graphs, in (ICALP 2003), vol. 2719 of Lecture Notes in Comput. Sci., Springer, Berlin, 2003, pp. 829-844.

[14] E. D. Demaine, M. Hajiaghayi, and D. M. Thilikos, Exponential speedup of fixed parameter algorithms on $K_{3,3}$-minor-free or $K_{5}$-minor-free graphs, in ISAAC 2002, Springer, Lecture Notes in Computer Science, Berlin, vol. 2518, 2002, pp. 262-273.

[15] J. Diaz, M. Serna, And D. M. Thilikos, Counting $H$-colorings of partial $k$-trees, Theoretical Computer Science, 281 (2002), pp. 291-309. 
[16] H. N. Djidjev and S. M. Venkatesan, Reduced constants for simple cycle graph separation, Acta Informatica, 34 (1997), pp. 231-243.

[17] R. G. Downey and M. R. Fellows, Parameterized complexity, Springer-Verlag, New York, 1999.

[18] D. Eppstein, Small maximal independent sets and faster exact graph coloring, in WADS 2001, vol. 2125 of Lecture Notes in Comput. Sci., Springer, Berlin, 2001, pp. $462-470$.

[19] U. FEIGE, Coping with the NP-hardness of the graph bandwidth problem, in SWAT 2000, vol. 1851 of Lecture Notes in Comput. Sci., Springer, Berlin, 2000, pp. 10-19.

[20] F. V. Fomin And D. M. Thilikos, Dominating sets in planar graphs: Branch-width and exponential speed-up, in SODA 2003, 2003, pp. 168-177.

[21] G. Gutin, T. Kloks, And C. M. Lee, Kernels in planar digraphs, in Optimization Online, Mathematical Programming Society, Philadelphia, 2001.

[22] M. M. Halldórsson And G. Kortsarz, Tools for multicoloring with applications to planar graphs and partial k-trees, Journal of Algorithms, 42 (2002), pp. 334-366.

[23] I. V. Hicks, Branch Decompositions and their applications, PhD thesis, Rice University, 2000.

[24] K. Jansen, M. Karpinski, A. Lingas, and E. Seidel, Polynomial time approximation schemes for Max-Bisection on planar and geometric graphs, in STACS 2001 (Dresden), vol. 2010 of Lecture Notes in Comput. Sci., Springer, Berlin, 2001, pp. 365375.

[25] I. Kanj And L. Perković, Improved parameterized algorithms for planar dominating set, in Mathematical Foundations of Computer Science-MFCS 2002, Springer, Lecture Notes in Computer Science, Berlin, vol. 2420, 2002, pp. 399-410.

[26] R. J. Lipton And R. E. TARJAn, A separator theorem for planar graphs, SIAM J. Appl. Math., 36 (1979), pp. 177-189.

[27] —_, Applications of a planar separator theorem, SIAM J. Comput., 9 (1980), pp. 615-627.

[28] N. Robertson and P. D. Seymour, Graph minors. X. Obstructions to treedecomposition, J. Combin. Theory Ser. B, 52 (1991), pp. 153-190.

[29] — Graph minors. XI. Circuits on a surface, J. Combin. Theory Ser. B, 60 (1994), pp. $72-106$. 
[30] N. Robertson, P. D. Seymour, and R. Thomas, Quickly excluding a planar graph, J. Combin. Theory Ser. B, 62 (1994), pp. 323-348.

[31] X. S. S. Nicoloso, Majid SARrafzadeh, On the sum coloring problem on interval graphs, Algorithmica, 23 (1999), pp. 109-126.

[32] P. D. Seymour and R. Thomas, Call routing and the ratcatcher, Combinatorica, 14 (1994), pp. 217-241.

[33] J. A. Telle and A. Proskurowski, Algorithms for vertex partitioning problems on partial k-trees, SIAM J. Discrete Math., 10 (1997), pp. 529-550.

[34] R. Thomas, Tree-decompositions of graphs. http://www.math.gatech.edu/ ${ }^{\sim}$ thomas/SLIDE/slide2.ps, p. 32.

[35] G. J. Woeginger, Exact algorithms for NP-hard problems: a survey, in Combinatorial Optimization--Eureka, You Shrink!, M. Junger, G. Reinelt, and G. Rinaldi, eds., Springer, LNCS 2570, Berlin, 2003, pp. p. 185-207. 


\section{A Appendix: Definitions of some problems}

A vertex cover $C$ of a graph is a set of vertices such that every edge of $G$ has at least one endpoint in $C$. A dominating set $D$ of a graph $G$ is a set of vertices such that every vertex outside $D$ is adjacent to a vertex of $D$. We denote the corresponding problems of finding minimum vertex cover and dominating set as Vertex Cover. and Dominating Set.

$(k, r)$-center is a natural generalization of dominating set. We define the $r$-neighborhood of a set $S \subseteq V(G)$, denoted by $N_{G}^{r}(S)$, to be the set of vertices of $G$ at distance at most $r$ from at least one vertex of $S$ if there exists a set $S$ of centers (vertices) of size at most $k$ such that $N_{G}^{r}(S)=V(G)$.

An independent set $I$ of a graph $G$ is a set of vertices such that no two vertices of $I$ are adjacent. The problem of computing maximum minimum independent set is INDEPENDENT SET.

LONGEST PATH (CYCLE) problem is to find a path (cycle) in a graph of the maximum length.

BISECTION is the problem of a partitioning the vertex set $V(G)$ of a graph $G$ into two parts $V_{1}$ and $V_{2}$, such that $\left|V_{1}\right|=\left|V_{2}\right|$ and the number of edges between $V_{1}$ and $V_{2}$ is minimal.

A set $S$ of vertices in a digraph $D=(V, A)$ is a kernel if $S$ is independent and every vertex in $V-S$ has an out-neighbor in $S$. We denote the corresponding decision problem as KERNEL.

Let $G=(V, E)$ be a graph and $x: V \rightarrow \mathbb{N}$ some function mapping vertices to nonnegative integers. Let also $p=\max _{v \in V} x(v)$. A non-preemptive multicoloring ${ }^{3}$ of $\mathrm{G}$ is an assignment $\psi$ mapping each vertex of $v$ to some set of $x(v)$ consecutive positive integers such that adjacent vertices receive non-intersecting sets. The sum of a multicoloring $\psi$ is equal to $\Sigma_{v \in V} \max _{i \in \psi(v)} i$. The makespan of a multicoloring $\psi$ is equal to $\max _{v \in V} \max _{i \in \psi(v)} i$.

\section{B Appendix: Creating majorities from slopes}

This part of Appendix is devoted to the complete proof of our main combinatorial result:

Theorem B.1. Let $G,|V(G)| \geq 5$, be a triangulated $\Sigma$-plane graph without multiple edges, drawn in $\Sigma$ along with its radial graph and let $k \geq 2$ be an integer. If there exists a uniform slope of order $k+1$ in $R_{G}$ then $G$ contains a majority of order $k$.

This section is devoted to the proof of Theorem B.1 and is organized as follows. We start with the definitions of the notions of variations and vibrations (Subsection B.1). Then we prove that any noose can be transformed, after applying to it a sequence of

\footnotetext{
${ }^{3}$ The multicoloring problem has numerous aplications in job scheduling on multiprocessor systems [22], traffic intersection control [9], compiler design and VLSI routing [31].
} 

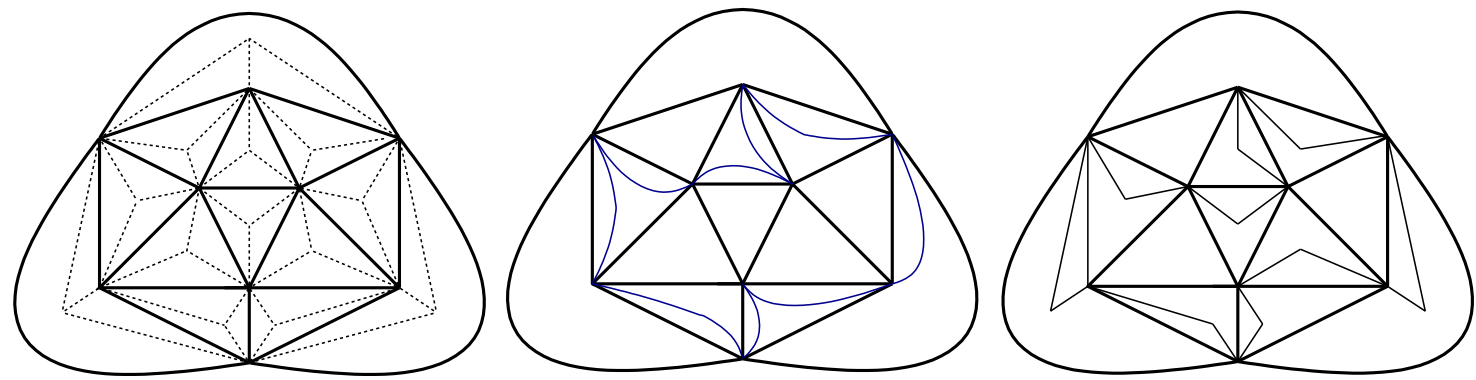

Figure 1: An example of a $\Sigma$-plane graph $G$ drawn i) with its radial $R_{G}$ ii) with a noose $S$ that is not a cycle of $R_{G}$ and with a noose $S^{\prime}$ that is a cycle of $R_{G}$ and a vibration of $S$.

variations, to a cycle of the radial graph (Subsection B.3). We also prove that the same type of representation via variations applies also to the $\Theta$-structures (Subsection B.4). That way, we are able to "translate" the slope axioms to majority ones. This requires a series of auxiliary results assuring that the basic topological properties involved in the majority axioms are invariants under vibrations (Subsection B.6). With all this knowledge on hands we proceed with the proof of the main result in Subsection B.7.

\section{B.1 Variations and vibrations}

If $G$ is a $\Sigma$-plane graph without loops or multiple edges and $S \subseteq \Sigma$ is an $I$-arc (simple closed curve) in $\Sigma$ then we use the notation $\kappa_{G}(S)=\left(v_{1}, \ldots, v_{|S \cap V(G)|}\right)$ for the ordering (cyclic ordering) of the vertex set $F \cap V(G)$ that represents the way the vertices of $G$ are met by $S$. Notice that $\kappa$ can be applied to both cycles and nooses but also to paths and lines. Especially for cycles and paths of graphs without multiple edges, we can directly represent them with the output of the function $\kappa$ (we will use the same notation for a cycle/path and the (cyclic) ordering of the vertices that it meets).

The basic idea of the proof is to correspond nooses of $G$ to cycles of $R_{G}$ and try to translate the slope axioms to majority axioms. Corresponding nooses to cycles is not direct as not every noose is a cycle of the radial graph (see Figure 1). To overcome this problem we need to introduce the concepts of variations and vibrations of nooses.

Let $S$ be one of the following structures in $G$ : a noose, a line, or a $\Theta$-structure. A variation of $S$ is the operation that transforms $S$ to another structure $S^{\prime}$ of the same type such that $\overline{\left(S \cup S^{\prime}\right)-\left(S \cap S^{\prime}\right)}$ is a noose of size 2 and one of the closed discs bounded by this noose, we denote this disc by $\operatorname{dif}\left(S, S^{\prime}\right)$, has the following two properties:

1. $\operatorname{dif}\left(S, S^{\prime}\right)-\mathbf{b d}\left(\operatorname{dif}\left(S, S^{\prime}\right)\right)$ contains no vertices of $G$,

2. $\operatorname{dif}\left(S, S^{\prime}\right)$ contains at most one edge of $G$.

If two structures $S_{1}$ and $S_{2}$ are variations each of the other, we denote it as $S_{1} \sim S_{2}$. If a structure $S^{\prime}$ is the result of a finite number of consecutive variations with $S$ as starting 


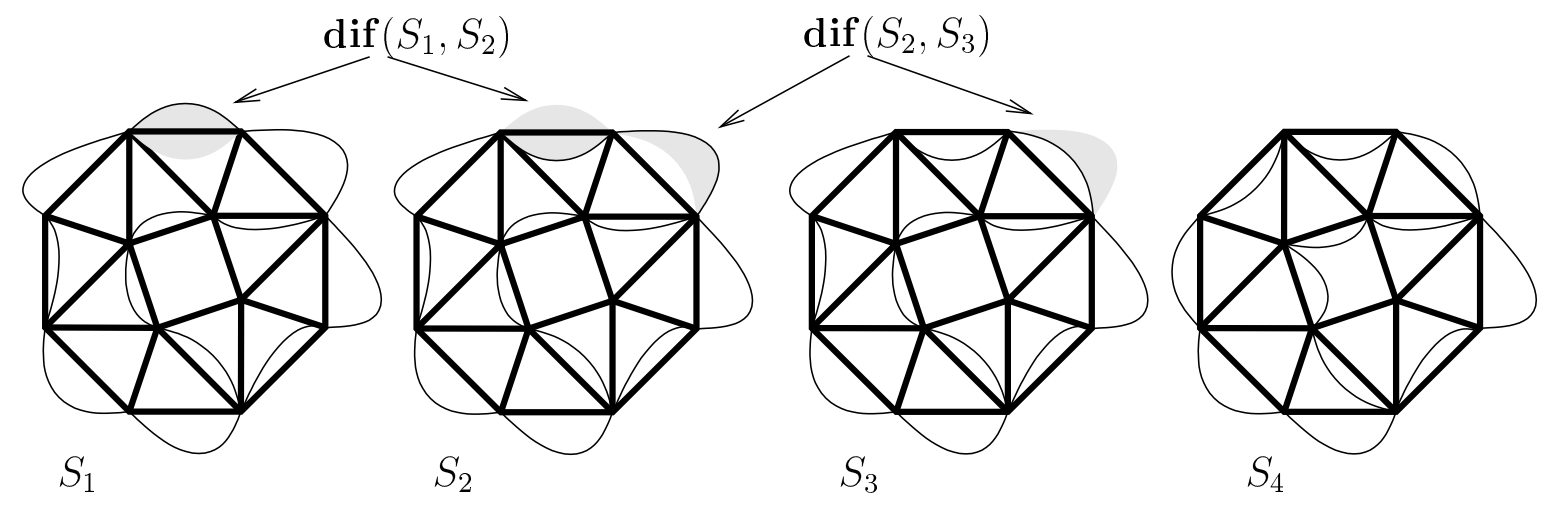

Figure 2: A $\theta$-structure $S_{1}$, a variation $S_{2}$ of $S_{1}$, a variation $S_{3}$ of $S_{2}$, and a vibration $S_{4}$ of all $S_{1}, S_{2}$, and $S_{3}$.
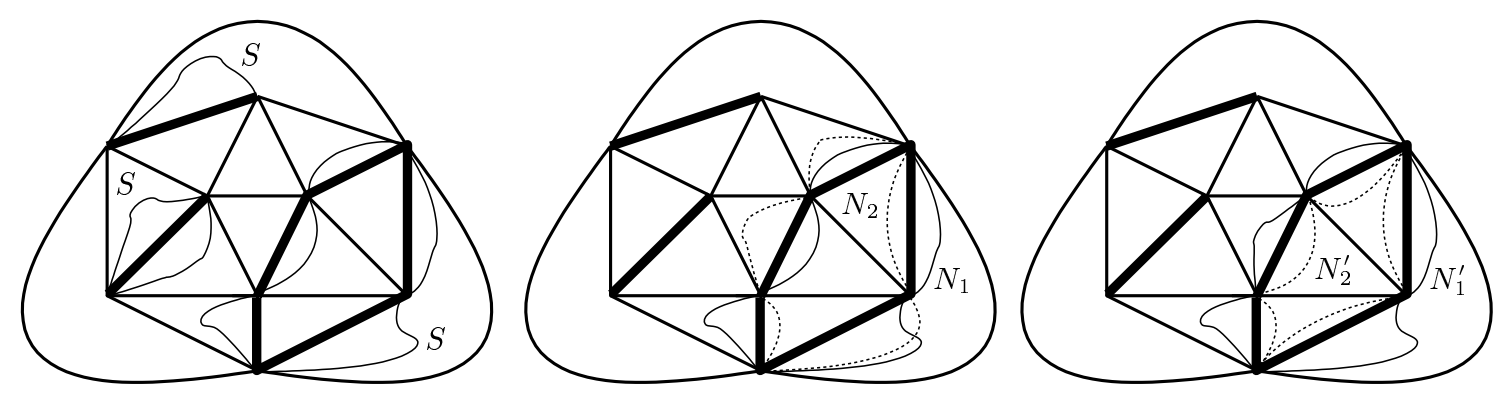

Figure 3: Examples of the proofs of Lemmata B.2 and B.3.

point, we call $S^{\prime}$ vibration of $S$ and we denote this fact as $S \sim^{*} S^{\prime}$. Notice that if $S \sim^{*} S^{\prime}$ then $V(G) \cap S=V(G) \cap S^{\prime}$ and $S$ and $S^{\prime}$ have the same length. In fact, it is easy to observe that if $N, N^{\prime}$ are nooses or lines where $N \sim^{*} N^{\prime}$ then $\kappa_{G}(N)=\kappa_{G}\left(N^{\prime}\right)$. Moreover, if $S=\left(L_{1}, L_{2}, L_{3}\right)$ and $S^{\prime}=\left(L_{1}^{\prime}, L_{2}^{\prime}, L_{3}^{\prime}\right)$ are $\Theta$-structures with $S \sim^{*} S^{\prime}$, then we order the elements of $S$ and $S^{\prime}$ such that for every $i, 1 \leq i<j \leq 3, L_{i} \cup L_{j} \sim^{*} L_{i}^{\prime} \cup L_{j}^{\prime}$. For examples of the notions of variation and vibration, see Figure 2.

\section{B.2 Corresponding nooses and lines to cycles and paths}

Lemma B.2. Let $G$ be a triangulated $\Sigma$-plane graph without multiple edges. If $S$ is a line or a noose of length 2 then exists a unique path $Q$ in $G$ such that $\kappa_{G}(S)=\kappa_{G}(Q)$. If $S$ is a noose of length $\geq 3$, then there exists a unique $Q$ in $G$ such that $\kappa_{G}(S)=\kappa_{G}(Q)$.

Proof. Let $\kappa_{G}(S)=\left(v_{0}, \ldots, v_{r-1}\right)$. We prove that for any $i=0, \ldots, r-2$, the vertices $v_{i}, v_{i+1} \in \kappa_{G}(S)$ are adjacent via only one edge (in case $S$ is a noose we take $i=0, \ldots, r-1$ and indices are taken modulo $r$ ). As $S$ is $G$-normal, the portion of $S$ that is between $v_{i}$ and $v_{i+1}$ should be a subset of some, say $r$, of the regions of $G$ (this region is not well 
defined only if $|V(G)|=3$ and, in this case, $r$ can be any region of $G$ ). Notice that $r$ is a triangle where $v_{i}, v_{i+1} \in \mathbf{b d}(r)$ and therefore $\left\{v_{i}, v_{i+1}\right\}$ is an edge of $G$. This edge is unique because $G$ does not have multiple edges (for an example, see the first graph of Figure 3).

Lemma B.3. Let $G$ be a triangulated $\Sigma$-planar graph without multiple edges and let $N_{1}, N_{2}$ be nooses of $G$ where $\left|N_{1}\right|,\left|N_{2}\right| \geq 3$. Then $\kappa_{G}\left(N_{1}\right)=\kappa_{G}\left(N_{2}\right)$ implies $N_{1} \sim^{*} N_{2}$.

Proof. Suppose that $N_{1}, N_{2}$ are nooses where $\left|N_{1}\right|,\left|N_{2}\right| \geq 3$ and $\kappa_{G}\left(N_{1}\right)=\kappa_{G}\left(N_{2}\right)$. By Lemma B.2, there is a unique cycle $C$ where $\kappa_{G}(C)=\kappa_{G}\left(N_{1}\right)$ and a unique cycle $C^{\prime}$ where $\kappa_{G}\left(C^{\prime}\right)=\kappa_{G}\left(N_{2}\right)$. As $\kappa_{G}(N)=\kappa_{G}\left(N^{\prime}\right)$ we have that $\kappa_{G}(C)=\kappa_{G}\left(C^{\prime}\right)$ and as $G$ does not have multiple edges, we have that $C=C^{\prime}$. We use the notation $C=\left(x_{0}, \ldots, x_{r-1}\right)$. For $j=1,2$, we define the function $\sigma_{j}$ corresponding to each edge $e_{i}=\left\{x_{i}, x_{i+1}\right\}$ of $C$ the unique line, $\sigma_{j}\left(e_{i}\right)$ in $\Sigma$ that is a subset of $N_{i}$ and has endpoints $x_{i}$ and $x_{i+1}$ (as $\left|N_{1}\right|,\left|N_{2}\right| \geq 3, \sigma_{j}$ is well defined). Let $\Delta_{1}, \Delta_{2}$ be the closed discs bounded by $C$ in $\Sigma$. We define

$$
\mathcal{D}_{j}=\left\{i \mid \sigma_{j}\left(e_{i}\right) \subseteq \Delta_{3-j}\right\}, j=1,2 .
$$

For $j=1,2$ we apply a sequence of variations on $N_{j}$ as indicated by the following routine. The target of this routine is to put the whole $N_{i}$ inside the closed disc $\Delta_{i}$.

1. If $\mathcal{D}_{j}$ is empty then stop and output $N_{j}$.

2. Pick an integer $i$ in $\mathcal{D}_{j}$.

3. Let $L$ be any line $L \subseteq \Sigma$ where $|L|=1, L \subseteq \Delta_{j}$, and $L \cap L_{j}=x_{i}, x_{i+1}$.

4. Set $N_{j} \leftarrow N_{j}-\sigma_{j}\left(e_{i}\right) \cup L$. (Notice that this is a variation operation on $N_{j}$.)

5. Recalculate $\sigma_{j}$ and $\mathcal{D}_{j}$. (Notice that now $i \notin \mathcal{D}_{j}$.)

6. Go to step 1.

For $j=1,2$, we call $N_{j}^{\prime}$ the resulting nooses and observe that $N_{j}^{\prime} \subseteq \Delta_{j}$ and $N_{j} \sim^{*} N_{j}^{\prime}$. We now apply the following sequence of variations on $N_{1}^{*}$ : For any $i=0, \ldots, r-1$, we set $N_{1}^{\prime}=N_{1}^{\prime}-\sigma_{1}\left(e_{i}\right) \cup \sigma_{2}\left(e_{i}\right)$. The resulting noose is $N_{2}$ and therefore, $N_{1}^{\prime} \sim^{*} N_{2}^{\prime}$. We conclude that $N_{1} \sim^{*} N_{2}$ and this completes the proof of the lemma (for an example, see the second and the third graph of Figure 3).

\section{B.3 Representing nooses by vibrations}

Observe that if $G$ is a $\Sigma$-plane graph drawn in $\Sigma$ along with its radial graph $R_{G}$ then any cycle of $R_{G}$ of length $2 k$ is a noose of length $k$. Any path of length $2 k$ in $R_{G}$ with both endpoints in $V(G)$ is a line in $G$ of length $k$. Notice that if $r$ is a region $R_{G}$ then $\mathbf{b d}(r)$ is a cycle of length 4 where $\bar{r}$ contains exactly one edge of $G$. Every edge $e$ of $G$ is contained 


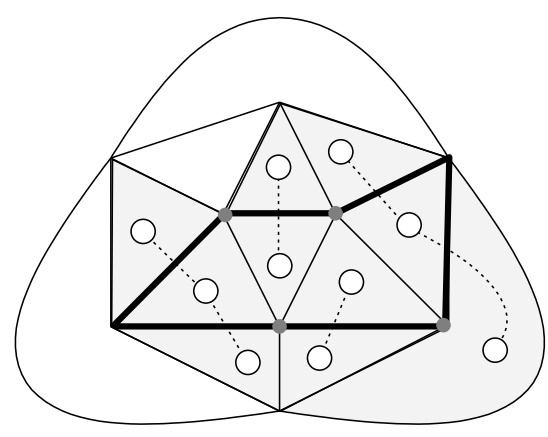

a.

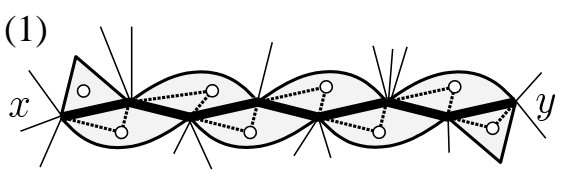

(2)

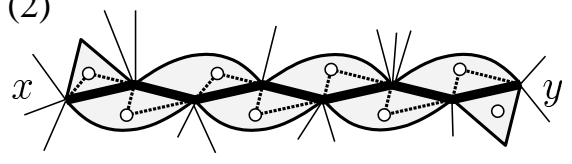

(1)

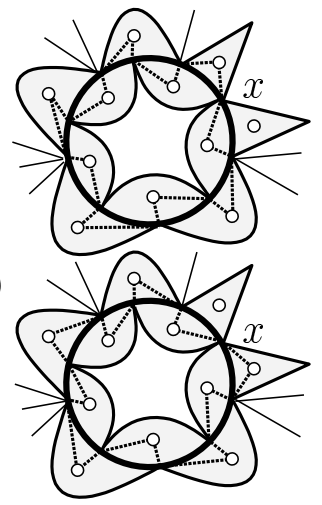

b.

Figure 4: a) If $F$ contains the edges of the "fat" cycle then the graph $H_{F}$ is the one formed by the dotted vertices and the white vertices. b) Examples of the constructions (1) and (2) of the proof of Lemma B.4 when generalized $(x)$-path is a $(x, y)$-paths and a cycle.

in $\bar{r}$ for some region $r$. From now on, we use the notation $\mathbf{r}_{e}$ to denote this region. If $T$ is a triangle of $G$ and $|V(G)| \geq 4$ then we use the notation $\mathbf{v}(T)$ for the unique vertex of $R_{G}$ that is adjacent in $R_{G}$ with all the vertices of $T$.

Let $G$ be a triangulated $\Sigma$-plane graph and let $F \subseteq E(G)$. We define the graph $H_{F}$ as the subgraph of a dual graph $G^{*}$ formed by edges $F^{*}$. In other words, its vertices are the triangles of $G$ that contain some edge in $F$ and two such triangles are connected by an edge if they have an edge of $F$ in common. To distinguish the vertices of $H_{F}$ from the vertices of the original graph we refer to the vertices of $H_{F}$ as to triangles.

Notice that, as $G$ is triangulated, the maximum degree of the vertices of $H_{F}$ is 3 (in the extreme case where the maximum degree is 3 we have that three of the edges in $F$ induce a triangle in $G$ ). This construction will be the basic common ingredient of the proofs of this and the next subsection. We call two triangles of degree 1 in $H_{F}$ irrelevant if they belong in different connected components of $H_{F}$.

We call a subgraph $P$ of a $\Sigma$-plane graph $G$ generalized $(x)$-path if either

- $P$ is a path with an extreme $x$, or

- it is a cycle of length $\geq 4$ passing through $x$ and such that there is no edge connecting the neighbors of $x$ in $P$.

Notice that the stressed cycle of the graph of Figure 4 is a generalized $(x)$-path iff $x$ is one of the grey vertices.

Lemma B.4. Let $G$ be a triangulated $\Sigma$-plane graph without multiple edges and where $|V(G)| \geq 4$, drawn in $\Sigma$ along with its radial graph $R_{G}$. Let also $P$ be a generalized $(x)$ path of $G$ with the property that $H_{E(P)}$ is connected. Let also $T$ be a triangle of degree 1 
in $H_{E(P)}$. Then there exists a generalized $(x)$-path $P_{R}$ in $R_{G}$ such that $\kappa_{G}\left(P_{R}\right)=\kappa_{G}(P)$ and $\mathbf{v}(T) \notin P_{R}$.

Proof. We use the notation $P=\left(x=v_{0}, \ldots, v_{r}=y\right), r \geq 1$ (in case $P$ is a cycle we have $x=y$ ). As $|V(G)| \geq 4$ and $G$ does not have multiple edges, the connectivity of $H_{E(P)}$ yields that $H_{E(P)}$ is a path whose extreme vertices are triangles of $G$. Each of these triangles has only one edge in common with $P$. Therefore we can denote them as $\left(a, v_{0}, v_{1}\right)$ and $\left(v_{r-1}, v_{r-2}, b\right)$ for some $a \neq v_{0}$ and $b \neq v_{d}$. Notice that, for $j=2, \ldots, r-2$ the edge $\left\{v_{j}, v_{j+1}\right\}$ is the common edge of the triangles $\left(v_{j-1}, v_{j}, v_{j+1}\right)$ and $\left(v_{j}, v_{j+1}, v_{j+2}\right)$ in $V(H)$. Moreover $\left\{v_{0}, v_{1}\right\}$ is the common edge of $\left(a, v_{0}, v_{1}\right)$ and $\left(v_{0}, v_{1}, v_{2}\right)$ and $\left\{v_{r-1}, v_{r}\right\}$ is the common edge of $\left(v_{r-2}, v_{r-1}, v_{r}\right)$ and $\left(v_{r-1}, v_{r}, b\right)$.

If $\left(b, v_{r-1}, v_{r}\right)=T$ we set

$$
\begin{aligned}
P_{R}= & \left(v_{0}, \mathbf{v}\left(a, v_{0}, v_{1}\right), v_{1}, \mathbf{v}\left(v_{0}, v_{1}, v_{2}\right), v_{2}, \mathbf{v}\left(v_{1}, v_{2}, v_{3}\right), \ldots\right. \\
& \left.\ldots, \mathbf{v}\left(v_{q-3}, v_{q-2}, v_{q-1}\right), v_{q-1}, \mathbf{v}\left(v_{r-2}, v_{r-1}, v_{r}\right), v_{r}\right)
\end{aligned}
$$

If $\left(a, v_{0}, v_{1}\right)=T$ we set

$$
\begin{aligned}
P_{R}= & \left(v_{0}, \mathbf{v}\left(v_{0}, v_{1}, v_{2}\right), v_{1}, \mathbf{v}\left(v_{1}, v_{2}, v_{3}\right), v_{2}, \ldots\right. \\
& \left.\ldots, v_{r-2}, \mathbf{v}\left(v_{r-2}, v_{r-1}, v_{r}\right), v_{r-1}, \mathbf{v}\left(b, v_{r-1}, v_{r}\right), v_{r}\right)
\end{aligned}
$$

In any case, we guarantee that we can choose a line $P_{R}$ that does not meet the vertex $\mathbf{v}(T)$. Observe that, by the construction of $P_{R}, \kappa_{G}\left(P_{R}\right)=\kappa_{G}(P)$ and the lemma follows. For examples of the above constructions see Figure 4 .

The next Lemma is a generalization of Lemma B.4 for the general case where $H_{E(P)}$ is not necessarily connected.

Lemma B.5. Let $G,|V(G)| \geq 4$, be a triangulated $\Sigma$-plane graph without multiple edges drawn in $\Sigma$ along with its radial graph $R_{G}$. Let also $P$ be a generalized $x$-path of $G$ and let $\mathcal{T}$ be a collection of mutually irrelevant degree one triangles in $V\left(H_{E(P)}\right)$. Then there exists a generalized $x$-path $P_{R}$ in $R_{G}$ such that $\forall_{T \in \mathcal{T}}, \mathbf{v}(T) \notin P_{R}$ and $\kappa_{G}\left(P_{R}\right)=\kappa_{G}(P)$.

Proof. Let $P_{1}, \ldots, P_{q}$ be the maximal sub-paths of $P$ with the property that $H_{E\left(P_{i}\right)}$ is connected. (When $P$ is a cycle these sub-paths still exist because $x$ belongs into two distinct degree one triangles of $H_{E(P)}$.) Notice that $\left\{P_{i} \mid i=1, \ldots, q\right\}$ is a partition of $P$ and assume that its indices order it into consecutive segments of $P$. We assume that the endpoints of $P_{i}$ are $a_{i}, b_{i}, 1 \leq i \leq q$ where $x=a_{1}, b_{1}=a_{2}, \ldots, b_{q-1}=a_{q}$, and $b_{q}=y$; the equalities follow from the maximality of each $P_{i}$ (when $P$ is a cycle, $x=y$ ). We denote as $H_{1}, \ldots, H_{q}$ the connected components of $H_{E(P)}$ indexed in a way that $H_{i}=H_{E\left(P_{i}\right)}$. Notice that $\left|\mathcal{T} \cap V\left(H_{i}\right)\right| \leq 1, i=1, \ldots, q$ (otherwise we should have two irrelevant degree one triangles in the same component of $H)$. If $\left|\mathcal{T} \cap V\left(H_{i}\right)\right|$ is non empty, then let $T_{i}$ be the unique triangle in it. Otherwise let $T_{i}$ be any of the triangles of $V\left(H_{i}\right)$ with degree 


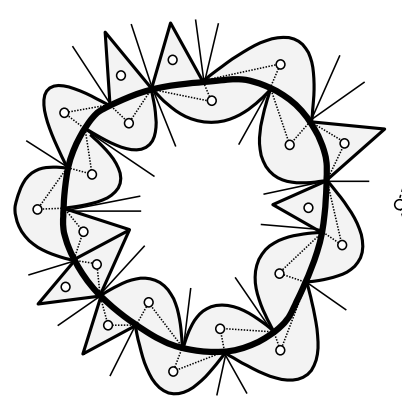

a.

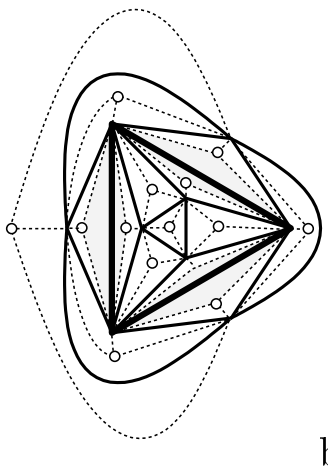

b.

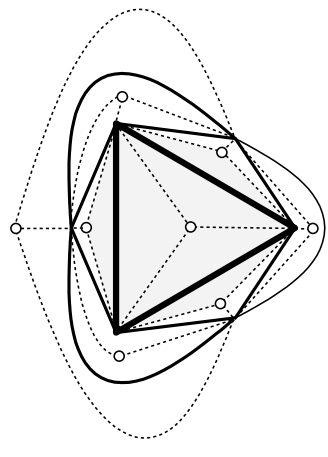$$
\text { . }
$$

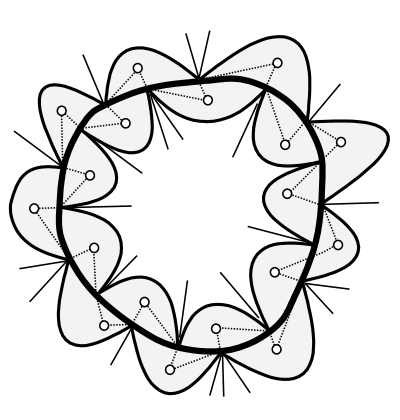

c.

Figure 5: a) An example of the proof of Lema B.5. b) Examples of the case $|C|=3$ of the proof of Lemma B.6. c) Example of the first case of the proof of Lemma B.6.

1 in $H_{i}$. We now apply Lemma B.4 for $H_{i}$ and $T_{i}$ and we get a path $P_{R}^{i}$ connecting $a_{i}$ and $b_{i}$ in $R_{G}$ and such that $\kappa_{G}\left(P_{R}^{i}\right)=\kappa_{G}\left(P^{i}\right)$ and $\mathbf{v}\left(T_{i}\right) \notin P_{R}^{i}$. We set $C_{R}=\bigcup_{i=1, \ldots, q} P_{R}^{i}$ and observe that, for any $T \in \mathcal{T}, \mathbf{v}\left(T_{i}\right) \notin P_{R}^{i}$. As none of the triangles in $H_{E(P)}$ belongs to two different connected components of $H_{E(P)}$, we have that $\kappa_{G}\left(P_{R}\right)=\kappa_{G}(P)$ and the lemma follows (for an example, see Figure 5.a.).

Lemma B.6. Let $G$ be a triangulated $\Sigma$-plane graph with $\geq 4$ vertices and without multiple edges, drawn in $\Sigma$ along with its radial graph $R_{G}$. Let also $C$ be a cycle in $G$ and $\mathcal{T}$ be an collection of mutually irrelevant degree one triangles in $H_{E(C)}$. Then there exists a cycle $C_{R}$ in $R_{G}$ such that $\kappa_{G}\left(C_{R}\right)=\kappa_{G}(C)$ and $\forall_{T \in \mathcal{T}}, \mathbf{v}(T) \notin C_{R}$.

Proof. If $|C|=3$, then we use the notation $C=(x, y, z)$ and we notice that

$$
\operatorname{bd}\left(\overline{\mathbf{r}}_{\{x, y\}\}} \cup \overline{\mathbf{r}}_{\{y, z\}} \cup \overline{\mathbf{r}}_{\{z, x\}}\right)
$$

is a subgraph of $R_{G}$ and contains as a subgraph at least one cycle $C_{R}$ of length 6 as required (it meets all the vertices of $C$, otherwise, $G$ should have a multiple edge - see also Figure 5.b).

Suppose now that $C=\left(x_{0}, \ldots, x_{r-1}, x_{0}\right), r \geq 4$. As $|C| \geq 4$, we have that all the vertices in $H_{E(C)}$ have degree at most 2 (otherwise $C$ is a triangle). We examine two cases:

Case 1: $H$ is a cycle of $r$ vertices. In this case we should have $\mathcal{T}=\emptyset$. Observe that

$$
C_{R}=\left(x_{0}, \mathbf{v}\left(x_{0}, x_{1}, x_{2}\right), x_{2}, \mathbf{v}\left(x_{1}, x_{2}, x_{3}\right), \ldots, x_{r-1}, \mathbf{v}\left(x_{r-1}, x_{0}, x_{1}\right), x_{0}\right)
$$

is the required cycle of $R_{G}$ (all indices are taken modulo $r$ ). For an example of this case, see Figure 5.c. 


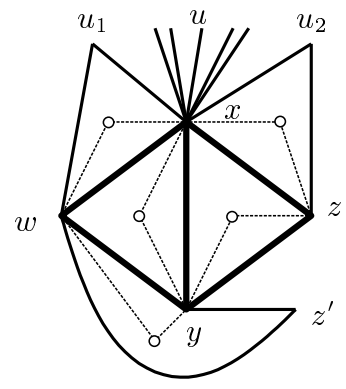

a.

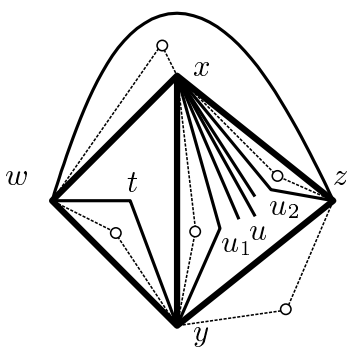

b.

Figure 6: The case $\left|P_{2} \cup P_{3}\right|=3$ and $\left|P_{1}\right|=2$ of the proof of Lemma B.8.

Case 2: All the connected components of $H$ are paths. In this case, there will exist a vertex $x \in C$ such that its neighbors in $C$ are not adjacent. Therefore $C$ is a generalized $(x)$-path, it is not a triangle, and by applying Lemma B.5 for $C$ and $\mathcal{T}$ the result follows.

The following lemma is the main conclusion of this subsection.

Lemma B.7. Let $G$ be a triangulated $\Sigma$-plane graph with $\geq 4$ vertices and without multiple edges, drawn in $\Sigma$ along with its radial graph $R_{G}$. Then any noose $N,|N| \geq 2$, of $G$ is a vibration of some of the cycles of $R_{G}$.

Proof. If $|N|=2$ then let $e$ be the unique edge connecting the extreme points of $N$ ( $e$ is unique because $G$ does not have multiple edges). We directly have that $\mathbf{b d}\left(\mathbf{r}_{e}\right)$ is a cycle of $R_{G}$ and it is easy to verify that it is also a vibration of $N$. Therefore, we may assume that $|N| \geq 3$. From Lemma B.2 there exist a unique cycle $C$ where $\kappa_{G}(C)=\kappa_{G}(N)$. From Lemma B.6, there exist a noose $C_{R}$ of $G$ where $\kappa_{G}\left(C_{R}\right)=\kappa_{G}(C)$. Notice that $C_{R}$ is a cycle of $R_{G}$ and, as $\kappa_{G}(N)=\kappa_{G}\left(C_{R}\right)$, from Lemma B.3, we conclude that $N \sim^{*} C_{R}$.

\section{B.4 Representing $\Theta$-structures by vibrations}

Let $N$ be a noose in $\Sigma$ and let $Q$ be a continuous subset of $\Sigma$ such that $N \cap Q=\emptyset$. then one of the discs bounded by $N$ does not contain points of $Q$. We call this disc by $Q$-avoiding disc bounded by $N$.

Lemma B.8. Let $G$ be a triangulated $\Sigma$-plane graph with $\geq 5$ vertices and without multiple edges, drawn in $\Sigma$ along with its radial graph $R_{G}$. Then for any three paths $P^{1}, P^{2}, P^{3}$ of $G$ that connect two vertices $x$ and $y$ and are otherwise disjoint, there exists three paths $P_{R}^{1}, P_{R}^{2}, P_{R}^{3}$ in $R_{G}$ that connect $x$ and $y$ and are otherwise disjoint and such that for any $i, 1 \leq i \leq 3, \kappa_{G}\left(P_{R}^{i}\right)=\kappa_{G}\left(P^{i}\right)$. 
Proof. We first examine the special case where some of $P_{1} \cup P_{2}, P_{1} \cup P_{3}$, or $P_{2} \cup P_{3}$ has length 3. W.l.o.g we assume that $\left|P_{2} \cup P_{3}\right|=3$ and, in particular we let $P_{2}=(x, y)$ and $P_{3}=(x, z, y)$. Notice that $\left|P_{1}\right| \geq 2$ because $G$ has not multiple edges. We examine two subcases:

$\left|P_{1}\right|=2$. We assume that $P_{1}=(x, w, y)$. We examine first the case where either $x$ or $y$ is connected with a vertex $u$ of the $\{x, y\}$-avoiding open disc $D$ bounded by $(x, z, y, w)$ (see Figure 6.a). W.l.o.g. assume that $x$ is adjacent to $u$ and let $\left(w, x, u_{1}\right)$ and $\left(z, x, u_{2}\right)$ be the regional triangles containing $\{w, x\}$ and $\{z, x\}$ where $u_{1}, u_{2} \in D$ (each of these two triangles can have $\{x, u\}$ as an edge). Let also $\left(w, y, z^{\prime}\right)$ be the regional triangle containing $\{w, y\}$ and such that $z^{\prime} \in \bar{D}$ (notice that $z$ and $z^{\prime}$ may be identical). Then we set $P_{R}^{1}=\left(x, \mathbf{v}\left(x, u_{1}, w\right), w, \mathbf{v}\left(y, w, z^{\prime}\right), y\right), P_{R}^{2}=(x, \mathbf{v}(x, w, y), y)$, and $P_{R}^{3}=\left(x, \mathbf{v}\left(z, u_{2}, x\right), z, \mathbf{v}(z, x, y), y\right)$. Observe that $P_{R}^{i}, i=1,2,3$ are paths and that for every $i, 1 \leq i \leq 3, \kappa_{G}\left(P_{R}^{i}\right)=\kappa_{G}\left(P^{i}\right)$.

In the remaining case, $w$ and $z$ are adjacent, and the triangles $(w, x, z)$ and $(w, y, z)$ are both regional (see Figure 6.b). Then, as $|V(G)| \geq 5$, there exist a vertex $u$ that is adjacent to either $x$ or $y$ and is included into either the $w$-avoiding open disc bounded by $(x, y, z)$ or into the $z$-avoiding open disc bounded by $(x, y, w)$. W.l.o.g. we assume that $u$ is adjacent to $x$ and that $x$ is included in the $w$-avoiding open disc $D$ bounded by $(x, y, z)$. Let $\left(x, u_{1}, y\right)$ and $\left(x, u_{2}, z\right)$ be the regional triangles containing $\{x, y\}$ and $\{x, z\}$ where $u_{1}, u_{2} \in D$ (each of these two triangles can have $\{x, u\}$ as an edge). Let also $(w, y, t)$ be a regional triangle containing $\{w, y\}$ where $t$ belongs in the $z$-avoiding open disc bounded by $(x, w, y)$. Then we set $P_{R}^{1}=(x, \mathbf{v}(x, w, z), w, \mathbf{v}(w, y, t), y), P_{R}^{2}=\left(x, \mathbf{v}\left(x, u_{1}, y\right), y\right)$ and $P_{R}^{3}=\left(x, \mathbf{v}\left(x, u_{2}, z\right), z, \mathbf{v}(w, z, y), y\right)$. Observe that for every $i, 1 \leq i \leq 3, P_{R}^{i}, i=1,2,3$ are paths and $\kappa_{G}\left(P_{R}^{i}\right)=\kappa_{G}\left(P^{i}\right)$.

$\left|P_{1}\right| \geq 3$. We assume that $P_{1}=\left(x=v_{0}, v_{1}, \ldots, v_{r-2}, v_{r}=y\right), r \geq 3$ and observe that $C=\left(v_{0}, v_{1}, \ldots, v_{r-1}, v_{r}\right)$ is a cycle of $G$ where $|C| \geq 4$. We call $D$ the $\{x, y\}$-avoiding closed disc bounded by $P_{1} \cup P_{3}$ in $\Sigma$. Let $T_{z}=(x, y, z)$. Also let $T_{x}=(x, z, a)$ be the unique regional triangle different than $(x, y, z)$ that contains $\{x, z\}$ and where $a \in D$ and let $T_{y}=(y, z, b)$ be the unique triangle different than $(x, y, z)$ that contains $\{y, z\}$ and where $b \in D$. We now construct the set $\mathcal{T}$ distinguishing 4 cases (see also Figure 7 ).

1. If $a \neq v_{1}$ and $b \neq v_{r-1}$ then we set $\mathcal{T}=\emptyset$.

2. If $a=v_{1}$ and $b \neq v_{r-1}$ then we have that $T_{x}$ is a triangle of degree 1 in $H_{E(C)}$ and we set $\mathcal{T}=\left\{T_{x}\right\}$.

3. If $a \neq v_{1}$ and $b=v_{r-1}$ then we have that $T_{y}$ is a triangle of degree 1 in $H_{E(C)}$ and we set $\mathcal{T}=\left\{T_{y}\right\}$.

4. If $a=v_{1}$ and $b=v_{r-1}$ then we have that both $T_{x}$ and $T_{y}$ are triangless of degree 1 in $H_{E(C)}$. As $|C| \geq 4$, any connected component of $H_{E(C)}$ has two triangles of degree 

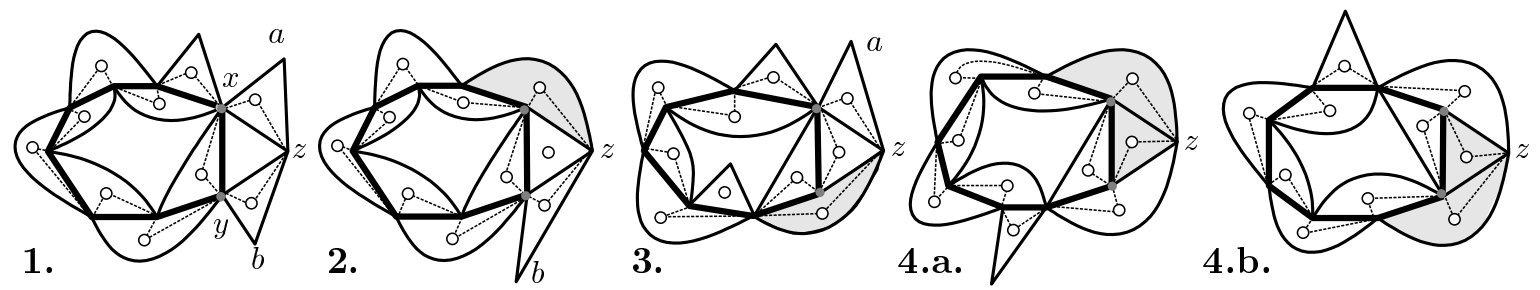

Figure 7: Examples of the proof of Lemma B.8 for the case where $\left|P_{2} \cup P_{3}\right|=3$ and $\left|P_{1}\right| \geq 3$ (subcases $1,2,3,4.1$ ).

1. This implies that either $\left\{T_{z}, T_{x}\right\}$ or $\left\{T_{z}, T_{y}\right\}$ is a collection of mutually irrelevant degree one triangles in $V\left(H_{E(C)}\right)$. We distinguish two subcases:

4.a. If $T_{z}$ and $T_{x}$ are irrelevant we set $\mathcal{T}=\left\{T_{z}, T_{x}\right\}$.

4.b. If $T_{z}$ and $T_{y}$ are irrelevant we set $\mathcal{T}=\left\{T_{z}, T_{y}\right\}$.

(If both pairs $T_{z}, T_{x}$ and $T_{z}, T_{y}$ are irrelevant we make an arbitrary choice.)

For any of the above cases we apply Lemma B.6 for $C$ and $\mathcal{T}$ and we get a cycle $C_{R}$ in $R_{G}$ where $\kappa_{G}\left(C_{R}\right)=\kappa_{G}(C)$. Clearly, $C_{R}$ is the union of two internally disjoint paths $P_{R}^{1}$ and $P_{R}^{2}$ that connect in $R_{G}$ the vertices $x$ and $y$. In cases $\mathbf{1}-\mathbf{3}$, we set $P_{R}^{3}=\left(x, \mathbf{v}\left(T_{x}\right), z, \mathbf{v}\left(T_{y}\right), y\right)$. In case $4 . \mathbf{a}$, we set $P_{R}^{3}=\left(x, \mathbf{v}\left(T_{x}\right), z, \mathbf{v}\left(T_{z}\right), y\right)$. In case 4.b, we set $P_{R}^{3}=\left(x, \mathbf{v}\left(T_{z}\right), z, \mathbf{v}\left(T_{y}\right), y\right)$. It is now easy to see that, in any case, for all $i, 1 \leq i \leq 3, \kappa_{G}\left(P_{R}^{i}\right)=\kappa_{G}\left(P^{i}\right)$. This completes the analysis of the special case.

Assume now that for all $i, j, 1 \leq i<j \leq 3,\left|P^{i} \cup P^{j}\right| \geq 4$. Let $P_{1}=\left(x, v_{1}, \ldots, v_{r-2}, y\right), P_{2}=$ $\left(x, u_{1}, \ldots, u_{s-2}, y\right)$ and $P_{3}=\left(x, w_{1}, \ldots, w_{t-2}, y\right)$. We consider the cycle $C=P^{1} \cup P^{2}$ and the path $P=P^{3}$. As $|C| \geq 4$ and $|P| \geq 3, V\left(H_{E(C)}\right)$ and $V\left(H_{E(P)}\right)$ can have at most 4 triangles in common that can be the triangles $A=\left(u_{1}, x, w_{1}\right), B=\left(v_{1}, x, w_{1}\right)$, $C=\left(u_{s-2}, y, w_{t-2}\right)$ and $D=\left(v_{r-2}, y, w_{t-2}\right)$. Our target will be to apply Lemmata B.5 and B.6 on $P$ and $C$ in order to construct a path $P_{R}$ and a cycle $C_{R}$ without common radial vertices. In order not to use the same interior vertices of $R_{G}$ two times we have to apply them with the restrictions imposed by suitably chosen collections $\mathcal{T}_{C}, \mathcal{I}_{P}$ of mutually irrelevant degree one triangles in $V\left(H_{E(C)}\right)$ and $V\left(H_{E(P)}\right)$ respectively. We set $\mathcal{C}=V\left(H_{E(C)}\right) \cap V\left(H_{E(P)}\right)$ and we distinguish the following cases (for examples, see Figures 8 and 9$)$.

1. $|\mathcal{C}|=0$. Then we set $\mathcal{T}_{C}=\mathcal{T}_{P}=\emptyset$.

2. $|\mathcal{C}|=1$. Then we set $\mathcal{T}_{C}=V\left(H_{E(C)}\right) \cap V\left(H_{E(P)}\right)$ and $\mathcal{T}_{P}=\emptyset$.

3. $|\mathcal{C}|=2$. Then we put in $\mathcal{T}_{C}$ one of the two elements of $\mathcal{C}$ and we put in $\mathcal{T}_{P}$ the other.

4. $|\mathcal{C}|=3$. Then we distinguish the following subcases: 


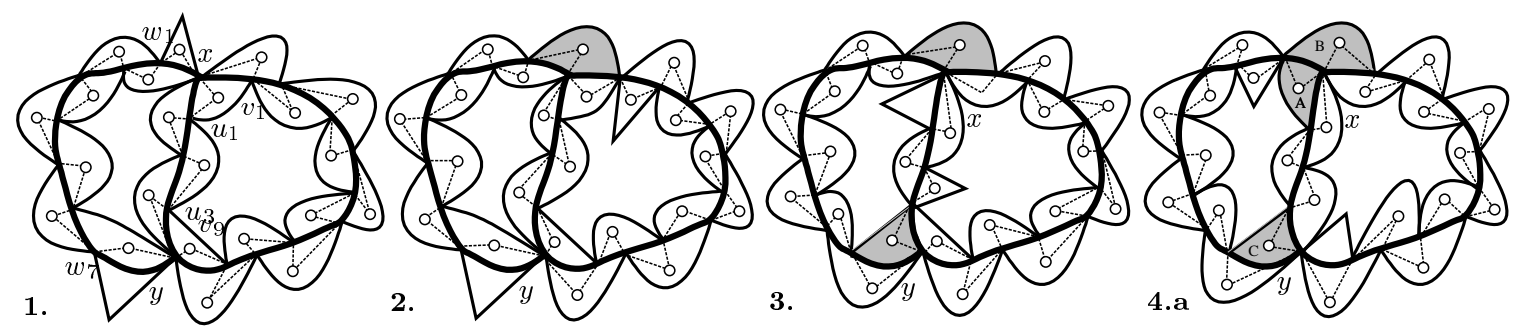

Figure 8: Examples of the proof of Lemma B.8 for the case where $\left|P_{2} \cup P_{3}\right|=3$ and $\left|P_{1}\right| \geq 3$ (subcases $\left.4.2,4.3,4.4,5\right)$.
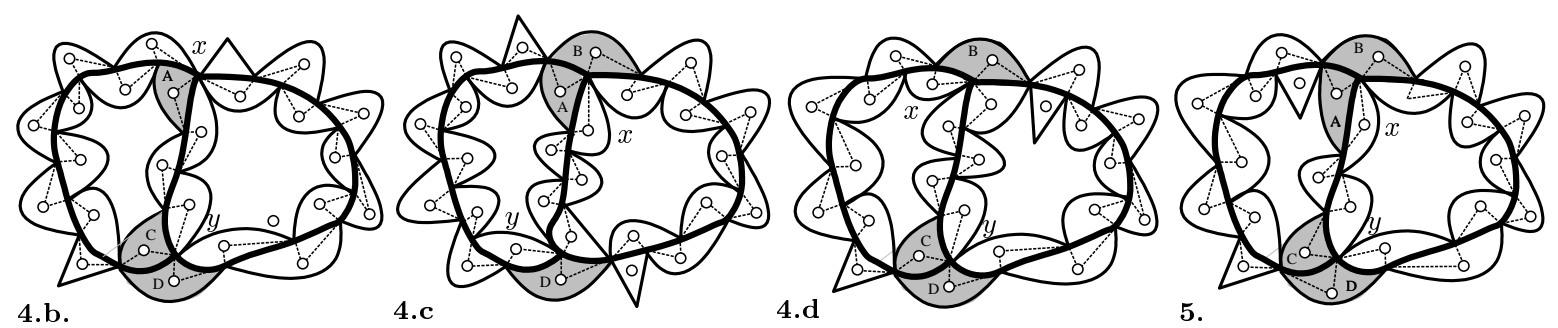

Figure 9: If $F$ contains the edges of the stressed cycle then the graph $H_{F}$ is the one formed by the dotted vertices and the white vertices.

4.a. if $\mathcal{C}=\{A, B, C\}$ then $\mathcal{T}_{C}=\{A\}$ and $\mathcal{T}_{P}=\{B, C\}$.

4.b. if $\mathcal{C}=\{A, C, D\}$ then $\mathcal{T}_{C}=\{C\}$ and $\mathcal{T}_{P}=\{A, D\}$.

4.c. if $\mathcal{C}=\{A, B, D\}$ then $\mathcal{T}_{C}=\{A\}$ and $\mathcal{T}_{P}=\{B, D\}$.

4.d. if $\mathcal{C}=\{B, C, D\}$ then $\mathcal{T}_{C}=\{C\}$ and $\mathcal{T}_{P}=\{B, D\}$.

5. $|\mathcal{C}|=4$. Then we set $\mathcal{T}_{C}=\{A, D\}$ and $\mathcal{T}_{P}=\{B, C\}$.

Notice that, in any of the above cases, the triangles in $\mathcal{T}_{C}$ and $\mathcal{T}_{P}$ are mutually irrelevant degree one triangles of $V\left(H_{E(C)}\right)$ and $V\left(H_{E(P)}\right)$ respectively. Therefore, we can apply Lemma B.5 for $P$ and $\mathcal{T}_{P}$ and Lemma B.6 for $C$ and $\mathcal{T}_{C}$ and construct the cycle $C_{R}$ and the path $P_{R}$ where $\kappa_{G}\left(C_{R}\right)=\kappa_{G}(C)$ and $\kappa_{G}\left(P_{R}\right)=\kappa_{G}(P)$. Notice that, in each case, the choice of $\mathcal{T}_{C}$ and $\mathcal{T}_{P}$ do not allow $C_{R}$ and $P_{R}$ to have common radial vertices. $C_{R}$ defines two paths $P^{1}$ and $P^{2}$ connecting $x$ and $y$ and if we set $P_{R}^{3}=P_{R}$ we have that $\kappa_{G}\left(P_{R}^{i}\right)=\kappa_{G}\left(P^{i}\right)$ for all $1 \leq i \leq 3$.

Let us remind that a $\Theta$-structure is non-trivial if at least two of its lines have length $\geq 2$.

Lemma B.9. Let $G$ be a triangulated $\Sigma$-plane graph with $\geq 5$ vertices and without multiple edges, drawn in $\Sigma$ along with its radial graph $R_{G}$. If $S=\left(L^{1}, L^{2}, L^{3}\right)$ is a non-trivial 
$\Theta$-structure of $G$, then there exist a non-trivial $\Theta$-structure $\left(P_{R}^{1}, P_{R}^{2}, P_{R}^{3}\right)$ of $G$ that is a vibration of $S$ where $P_{R}^{1}, P_{R}^{2}$ and $P_{R}^{3}$ are paths of $R_{G}$.

Proof. We apply Lemma B.2 for the noose $N=L^{1} \cup L^{2}$ and we get a cycle $C$ of $G$ where $\kappa_{G}(C)=\kappa_{G}(N)$. This cycle defines two internally disjoint paths $P^{1}$ and $P^{2}$ between $x$ and $y$ in $G$ where $\kappa_{G}\left(P^{i}\right)=\kappa_{G}\left(L^{i}\right), i=1,2$. Applying now again Lemma B.2 for the line $L_{3}$, we get a path $P^{3}$ between $x$ and $y$ in $G$ where $\kappa_{G}\left(P^{3}\right)=\kappa_{G}\left(L^{3}\right)$. We now apply Lemma B.8 on $P^{i}, i=1,2,3$ and get three internally disjoint paths $P_{R}^{1}, P_{R}^{2}, P_{R}^{3}$ of $R_{G}$ that connect $x$ an $y$ and such that for each $i, 1 \leq i \leq 3, \kappa_{G}\left(P_{R}^{i}\right)=\kappa_{G}\left(P^{i}\right)$. Resuming the previous equalities we get $\kappa_{G}\left(P_{R}^{i}\right)=\kappa_{G}\left(L^{i}\right), 1 \leq i \leq 3$. Notice that $\left(P_{R}^{1}, P_{R}^{2}, P_{R}^{3}\right)$ is a non-trivial $\Theta$-structure in $G$. In what remains we will show that it is also a vibration of $\left(L^{1}, L^{2}, L^{3}\right)$. Notice that $\kappa_{G}\left(P_{R}^{1} \cup P_{R}^{2}\right)=\kappa_{G}\left(L^{1} \cup L^{2}\right)$ and applying Lemma B.3 we have that $P_{R}^{1} \cup P_{R}^{2} \sim^{*} L^{1} \cup L^{2}$ and this, in turn, implies that $P_{R}^{1} \sim^{*} L^{1}$ and $P_{R}^{2} \sim^{*} L^{2}$. Notice now that $P_{R}^{2} \cup L^{3}$ is a noose of $G$. Recall that $\kappa_{G}\left(P_{R}^{3}\right)=\kappa_{G}\left(L^{3}\right)$ which implies that $\kappa_{G}\left(L^{2} \cup P_{R}^{3}\right)=\kappa_{G}\left(L^{2} \cup L^{3}\right)$. From Lemma B.3 we have that $L^{2} \cup P_{R}^{3} \sim^{*} L^{2} \cup L^{3}$ and this, in turn, implies that $P_{R}^{3} \sim^{*} L^{3}$. Therefore, $\left(P_{R}^{1}, P_{R}^{2}, P_{R}^{3}\right)$ is a vibration of $\left(L^{1}, L^{2}, L^{3}\right)$.

\section{B.5 A topological property of $\Theta$-structures}

Lemma B.10. Let $S=\left(L_{1}, L_{2}, L_{3}\right)$ and $S^{\prime}=\left(L_{1}^{\prime}, L_{2}, L_{3}\right)$ be two non-trivial $\Theta$-structures of some $\Sigma$-plane graph $G$ where $S \sim S^{\prime}$. Then, for one, say $D^{*}$, of the closed discs bounded by $L_{2} \cup L_{3}$, holds that $D^{*} \cap \operatorname{dif}\left(S, S^{\prime}\right) \subseteq L_{2} \cap L_{3}$.

Proof. Let $\{x, y\}=L_{2} \cap L_{3}$. Let also $L$ and $L^{\prime}$ be the length-1 lines comprising the length-2 noose $\left(S \cup S^{\prime}\right)-\left(S \cap S^{\prime}\right)=L \cup L^{\prime}$, assuming that $L \subseteq L_{1}$ and $L^{\prime} \subseteq L_{1}^{\prime}$. In the case analysis that follows, we will define a disc $D^{*}$ bounded by $L_{2} \cup L_{3}$ and we will show that $L \cup L^{\prime} \subseteq \overline{\Sigma-D^{*}}$.

Case 1. If $\left|L_{1}\right|,\left|L_{1}^{\prime}\right| \geq 2$, we can choose a vertex $v \in\left(L \cup L^{\prime}\right) \cap V(G)$ that is different that $x$ and $y$. Therefore $v \notin L_{2} \cup L_{3}$ and we can define $D^{*}$ as the closed disc bounded by $L_{2} \cup L_{3}$ that does not contain $v$. Notice that $L_{1} \cup L_{1}^{\prime}$ contains at most one point in common with $L_{2} \cup L_{3}=\mathbf{b d}\left(D^{*}\right)=\mathbf{b d}\left(\overline{\Sigma-D^{*}}\right)$. We need the following topological fact.

Fact 1 . Let $\Delta$ be a closed disc on a sphere $\Sigma$ and let $N$ be a simple closed curve where $N \cap \mathbf{b d}(\Delta)$ is either empty or is just a point $x$. Then $(\Delta-\mathbf{b d}(\Delta)) \cap N \neq \emptyset$ implies $N \subseteq \Delta$.

As $\left(\Sigma-D^{*}\right) \cap\left(L \cup L^{\prime}\right) \neq \emptyset$, we apply the fact for $L \cup L^{\prime}$ and $\overline{\Sigma-D^{*}}$, obtaining $L \cup L^{\prime} \subseteq \overline{\Sigma-D^{*}}$.

Case 2. $\left|L_{1}\right|,\left|L_{1}^{\prime}\right|=1$. Notice that, then, $\left|L_{2}\right|,\left|L_{3}\right| \geq 2$. Notice that $L_{1}-\{x, y\}$ cannot have common points with the noose $L_{2} \cup L_{3}$. Therefore it will be a subset of some of the closed discs bounded by $L_{2} \cup L_{3}$. Notice also that the same holds for $L_{1}^{\prime}$. Observe now that $L_{1}-\{x, y\}, L_{1}^{\prime}-\{x, y\}$ cannot be subsets of different discs bounded by the noose $L_{2} \cup L_{3}$ because then each of the discs bounded by the noose $L_{1} \cup L_{1}^{\prime}$ should contain a 


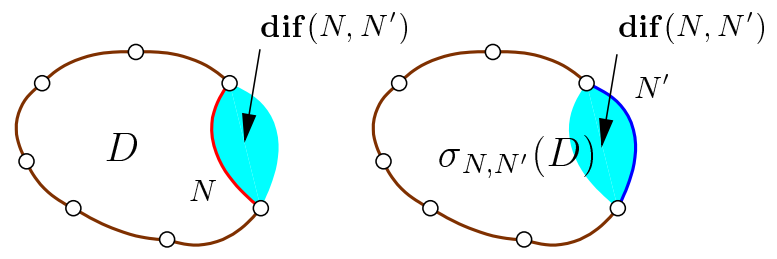

Figure 10: An example of the application of the function dif.

vertex of $G$. Let $D^{*}$ be the disc containing none of $L_{1}-\{x, y\}, L_{1}^{\prime}-\{x, y\}$. This means that the noose $L_{1} \cup L_{1}^{\prime}$ is a subset of $\overline{\Sigma-D^{*}}$. As $L_{1}=L$ and $L_{1}^{\prime}=L^{\prime}$, we have that $L_{1} \cup L_{1}^{\prime} \subseteq \overline{\Sigma-D^{*}}$.

Here is the second topological property we use in our proof.

Fact 2. Let $\Delta$ be a closed disc on a sphere $\Sigma$ and let $N$ be a simple closed curve where $N \subseteq \Delta$. Then some of the closed discs bounded by $N$ will be a subset of $\Delta$.

Let $A$ and $A^{\prime}$ be the discs bounded by $L_{1} \cup L_{1}^{\prime}$. By Fact 2, one, say $A$, of $A, A^{\prime}$ should be a subset of $\overline{\Sigma-D^{*}}$. Notice that $A$ should be $\operatorname{dif}\left(S, S^{\prime}\right)$, otherwise $A=\overline{\Sigma-\operatorname{dif}\left(S, S^{\prime}\right)}$ and as $A \subseteq \overline{\Sigma-D^{*}}$ we have that $\overline{\Sigma-\operatorname{dif}\left(S, S^{\prime}\right)} \subseteq \overline{\Sigma-D^{*}} \Rightarrow D^{*} \subseteq \operatorname{dif}\left(S, S^{\prime}\right)$. Hence $D^{*} \cap V(G) \subseteq \operatorname{dif}\left(S, S^{\prime}\right) \cap V(G)=\{x, y\}$ a contradiction as $\left|\left(D^{*} \cap V(G)\right)-\{x, y\}\right| \geq 1$

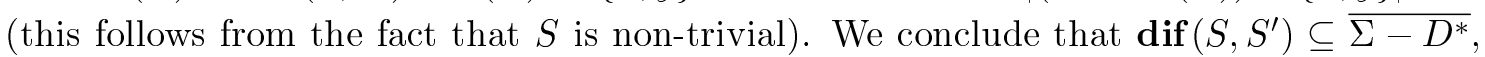
therefore $\operatorname{dif}\left(S, S^{\prime}\right)-\mathbf{b d}\left(\operatorname{dif}\left(S, S^{\prime}\right)\right) \subseteq \Sigma-D^{*} \Rightarrow\left(\operatorname{dif}\left(S, S^{\prime}\right)-\operatorname{bd}\left(\operatorname{dif}\left(S, S^{\prime}\right)\right) \cap D^{*}=\emptyset\right.$. As bd $\left(\operatorname{dif}\left(S, S^{\prime}\right)\right)=L_{1} \cup L_{1}^{\prime}$, we have that $\mathbf{b d}\left(\operatorname{dif}\left(S, S^{\prime}\right)\right) \cap D^{*}=\left(L_{1} \cup L_{1}^{\prime}\right) \cap D^{*} \subseteq\{x, y\}$ and the proof is complete.

\section{B.6 Vibration invariants of $\Theta$-structures}

Let $N, N^{\prime}$ be two nooses of some $\Sigma$-plane graph $G$. Let $N \sim N^{\prime}$ and let $\mathcal{D}=\left\{D_{1}, D_{2}\right\}$ and $\mathcal{D}^{\prime}=\left\{D_{1}^{\prime}, D_{2}^{\prime}\right\}$ be the closed discs bounded by $N$ and $N^{\prime}$ respectively. We set up a bijection $\sigma_{N, N^{\prime}}: \mathcal{D} \rightarrow \mathcal{D}^{\prime}$ such that if $D \in \mathcal{D}$ then

$$
\sigma_{N, N^{\prime}}(D)= \begin{cases}\overline{D-\operatorname{dif}\left(N, N^{\prime}\right)} & \text { if } \operatorname{dif}\left(N, N^{\prime}\right) \subseteq D \\ D \cup \operatorname{dif}\left(N, N^{\prime}\right) & \text { if } \operatorname{dif}\left(N, N^{\prime}\right) \nsubseteq D\end{cases}
$$

Also, for notational convenience, we enhance the definition of $\sigma$ so that $\sigma_{N, N}(D)=D$. It is easy to verify that $\sigma_{N, N^{\prime}}=\sigma_{N^{\prime}, N}^{-1}$ (for an example, see Figure 10).

Let $N$ and $N^{\prime}$ be nooses where $N \sim^{*} N^{\prime}$. Then if $N=N_{0} \sim N_{1} \sim \cdots \sim N_{r-1} \sim N_{r}=$ $N^{\prime}$, we define $\sigma_{N, N^{\prime}}^{*}=\sigma_{N_{0}, N_{1}} \circ \sigma_{N_{1}, N_{2}} \circ \cdots \circ \sigma_{N_{r-1}, N_{r}}$. Notice that $\sigma_{N, N^{\prime}}^{*}$ is well defined as it does not depend on the way $N$ is transformed to $N^{\prime}$ (however we stress that this fact is not used in our proofs). Again it follows that $\sigma_{N, N^{\prime}}^{*}=\sigma_{N^{\prime}, N}^{*-1}$.

The following lemma is a direct consequence of the fact that $\operatorname{dif}\left(N, N^{\prime}\right)$ does not contain vertices that are not met by both $N$ and $N^{\prime}$. 
Lemma B.11. Let $N_{1}, N_{2}$ be nooses of $G$ where $N_{1} \sim^{*} N_{2}$. If $D$ is some disc bounded by $N_{1}$ then $V(G) \cap \sigma_{N_{1}, N_{2}}^{*}(D)=V(G) \cap D$.

We need the following lemma.

Lemma B.12. Let $G$ be a $\Sigma$-plane graph and $S=\left(L_{1}, L_{2}, L_{3}\right)$ and $S^{\prime}=\left(L_{1}^{\prime}, L_{2}^{\prime}, L_{3}^{\prime}\right)$ be non-trivial $\Theta$-structures in $G$ where $S \sim^{*} S^{\prime}$. If $D$ is a closed disc bounded by the noose $L_{1} \cup L_{2}$ and $L_{3} \subseteq D$ then $L_{3}^{\prime} \subseteq \sigma_{L_{1} \cup L_{2}, L_{1}^{\prime} \cup L_{2}^{\prime}}^{*}(D)$.

Proof. It is sufficient to prove the statement of the lemma only for the case $L_{3}^{\prime} \subseteq \sigma_{L_{1} \cup L_{2}, L_{1}^{\prime} \cup L_{2}^{\prime}}(D)$. (Using this case as an induction assumption, one can prove the lemma by making use of induction on the number of variations required in order to transform $S$ to $S^{\prime}$.)

We set $\{x, y\}=L_{1} \cap L_{2} \cap L_{3}$. We also set $\Delta=\operatorname{dif}\left(S, S^{\prime}\right)$ and notice that a variation affects only one of the lines in $S$. Therefore, we can distinguish the following cases.

Case 1. $L_{2} \cup L_{3}=L_{2}^{\prime} \cup L_{3}^{\prime}$. Then $\Delta=\operatorname{dif}\left(L_{1} \cup L_{2}, L_{1}^{\prime} \cup L_{2}^{\prime}\right)$.

Subcase 1.a. If $\Delta \nsubseteq D$ then $\sigma_{L_{1} \cup L_{2}, L_{1}^{\prime} \cup L_{2}^{\prime}}(D)=D \cup \Delta$. Therefore, $L_{3}^{\prime}=L_{3} \subseteq D \subseteq$ $D \cup \Delta=\sigma_{L_{1} \cup L_{2}, L_{1}^{\prime} \cup L_{2}^{\prime}}(D)$.

Subcase 1.b. If $\Delta \subseteq D$, apply Lemma B.10 on $S$ and $S^{\prime}$ and let $D_{2,3}$ be the closed disc bounded by $L_{2} \cup L_{3}$ where $D_{2,3} \cap \Delta \subseteq\{x, y\}$. As $\left(L_{1}-\{x, y\}\right) \cap \Delta \neq \emptyset$, it implies that $L_{1}-\{x, y\} \subseteq \Sigma-D_{2,3}$. This means that $D_{2,3} \subseteq D$. We now have $D_{2,3}-\{x, y\} \subseteq$ $D_{2,3}-\left(D_{2,3} \cap \Delta\right)=D_{2,3}-\Delta \subseteq D-\Delta$. Therefore, $L_{3} \subseteq D_{2,3}=\overline{D_{2,3}-\{x, y\}} \subseteq \overline{D-\Delta}=$ $\sigma_{L_{1} \cup L_{2}, L_{1}^{\prime} \cup L_{2}^{\prime}}(D)$.

Case 2. $L_{1} \cup L_{3}=L_{1}^{\prime} \cup L_{3}^{\prime}$. This case is symmetric to the Case 1 .

Case 3. $L_{1} \cup L_{2}=L_{1}^{\prime} \cup L_{2}^{\prime}$. Again we apply Lemma B.10 on $S$ and $S^{\prime}$ and let $D_{1,2}$ be the disc bounded by $L_{1} \cup L_{2}$ where $D_{1,2} \cap \Delta \subseteq\{x, y\}$. As $\left(L_{3}-\{x, y\}\right) \cap \Delta \neq \emptyset$, we imply that $L_{3}-\{x, y\} \subseteq \Sigma-D_{1,2}$. Applying the same argument for $L_{3}^{\prime}$ we get $L_{3}^{\prime}-\{x, y\} \subseteq \Sigma-D_{1,2}$. Therefore, $L_{3}$ and $L_{3}^{\prime}$ are both included in the same disc bounded by $L_{1} \cup L_{2}$. As $L_{3} \subseteq D$ we conclude $L_{3}^{\prime} \subseteq D=\sigma_{L_{1} \cup L_{2}, L_{1}^{\prime} \cup L_{2}^{\prime}}(D)$.

Lemma B.13. Let $G$ be a $\Sigma$-plane graph and $S=\left(L_{1}, L_{2}, L_{3}\right)$ and $S^{\prime}=\left(L_{1}^{\prime}, L_{2}^{\prime}, L_{3}^{\prime}\right)$ be non-trivial $\Theta$-structures in $G$ where $S \sim^{*} S^{\prime}$. If $D_{1,2}$ is a closed disc bounded by the noose $L_{1} \cup L_{2}$ and $D_{1,3}$ is a closed disc bounded by the noose $L_{1} \cup L_{3}$ such that $D_{1,3} \subseteq D_{1,2}$ then $\sigma_{L_{3} \cup L_{3}, L_{1}^{\prime} \cup L_{3}^{\prime}}^{*}\left(D_{1,3}\right) \subseteq \sigma_{L_{1} \cup L_{2}, L_{1}^{\prime} \cup L_{2}^{\prime}}^{*}\left(D_{1,2}\right)$.

Proof. As in the previouse lemma, it is sufficient to prove only the case $S \sim S^{\prime}$. (And then use the induction on the number of variations required in order to transform $S$ to $S^{\prime}$.)

We set $\{x, y\}=L_{1} \cap L_{2} \cap L_{3}$. We also set $\Delta=\operatorname{dif}\left(S, S^{\prime}\right)$ and notice that a variation affects only one of the lines in $S$. Therefore, we can distinguish the following cases.

Case 1. $L_{2} \cup L_{3}=L_{2}^{\prime} \cup L_{3}^{\prime}$. Notice that $\Delta=\operatorname{dif}\left(L_{1} \cup L_{3}, L_{1}^{\prime} \cup L_{3}^{\prime}\right)$ 
Subcase 1.a. If $\Delta \nsubseteq D_{1,2}$ then, from, $D_{1,3} \subseteq D_{1,2}$ we also have that $\Delta \nsubseteq D_{1,3}$. Therefore, $\sigma_{L_{1} \cup L_{2}, L_{1}^{\prime} \cup L_{2}^{\prime}}\left(D_{1,2}\right)=D_{1,2} \cup \Delta, \sigma_{L_{1} \cup L_{3}, L_{1}^{\prime} \cup L_{3}^{\prime}}\left(D_{1,3}\right)=D_{1,3} \cup \Delta$ and the required relation follows as $D_{1,3} \subseteq D_{1,2}$.

Subcase 1.b. If $\Delta \subseteq D_{1,2}$ we apply Lemma B.10 on $S$ and $S^{\prime}$ and let $D_{2,3}$ be the disc bounded by $L_{2} \cup L_{3}$ where $D_{2,3} \cap \Delta \subseteq\{x, y\}$. As $\left(L_{1}-\{x, y\}\right) \cap \Delta \neq \emptyset$, we imply that $L_{1}-\{x, y\} \subseteq \Sigma-D_{2,3}$. This means that $D_{2,3} \subseteq D_{1,2}$. Combining this with the fact that $D_{1,3} \subseteq D_{1,2}$, we have that $D_{1,2}=D_{1,3} \cup D_{2,3}$. So, we can assume that $D_{1,2}-D_{2,3} \subseteq D_{1,3}$. Notice that $\Delta-\{x, y\} \subseteq \Delta-\left(D_{2,3} \cap \Delta\right)=\Delta-D_{2,3} \subseteq D_{1,2}-D_{2,3} \subseteq D_{1,3}$. As also $\{x, y\} \subseteq D_{1,3}$, we have that $\Delta \subseteq D_{1,3}$ and therefore $\sigma_{L_{1} \cup L_{3}, L_{1}^{\prime} \cup L_{3}^{\prime}}\left(D_{1,3}\right)=\overline{D_{1,3}-\Delta}$. Moreover, $\sigma_{L_{1} \cup L_{2}, L_{1}^{\prime} \cup L_{2}^{\prime}}\left(D_{1,2}\right)=\overline{D_{1,2}-\Delta}$ and the result follows as $\overline{D_{1,3}-\Delta} \subseteq \overline{D_{1,2}-\Delta}$.

Case 2. $L_{1} \cup L_{2}=L_{1}^{\prime} \cup L_{2}^{\prime}$. Notice that $\Delta=\operatorname{dif}\left(L_{1} \cup L_{3}, L_{1}^{\prime} \cup L_{3}^{\prime}\right)$.

Observe that in this case the variation does not affect the noose $L_{1} \cup L_{2}$. Therefore, $\sigma_{L_{1} \cup L_{2}, L_{1}^{\prime} \cup L_{2}^{\prime}}\left(D_{1,2}\right)=D_{1,2}$. In both subcases that follow, our target will be to prove that $D_{1,2} \supseteq \sigma_{L_{1} \cup L_{3}, L_{1}^{\prime} \cup L_{3}^{\prime}}\left(D_{1,3}\right)$.

Subcase 2.a. If $\Delta \nsubseteq D_{1,3}$, we apply Lemma B.10 on $S$ and $S^{\prime}$ and let $D^{*}$ be a disc bounded by $L_{1} \cup L_{2}$ where $D^{*} \cap \Delta \subseteq\{x, y\}$. As $\left(L_{3}-\{x, y\}\right) \cap \Delta \neq \emptyset$, we imply that $L_{3}-\{x, y\} \subseteq \Sigma-D^{*}$. As $L_{3} \subseteq D_{1,2}$, we get that $D^{*}=\Sigma-D_{1,2}$. Combining this with $D^{*} \cap \Delta \subseteq\{x, y\}$ we take $\Delta \subseteq D_{1,2}$. Therefore $\sigma_{L_{1} \cup L_{3}, L_{1}^{\prime} \cup L_{3}^{\prime}}\left(D_{1,3}\right)=D_{1,3} \cup \Delta \subseteq D_{1,2} \cup \Delta \subseteq$ $D_{1,2}$.

Subcase 2.b. If $\Delta \subseteq D_{1,3}$ then $\sigma_{L_{1} \cup L_{3}, L_{1}^{\prime} \cup L_{3}^{\prime}}\left(D_{1,3}\right)=\overline{D_{1,3}-\Delta} \subseteq D_{1,3} \subseteq D_{1,2}$.

Case 3. $L_{1} \cup L_{3}=L_{1}^{\prime} \cup L_{3}^{\prime}$. Notice that $\Delta=\operatorname{dif}\left(L_{1} \cup L_{2}, L_{1}^{\prime} \cup L_{2}^{\prime}\right)$.

Observe that in this case the variation does not affect the noose $L_{1} \cup L_{3}$. Therefore, $\sigma_{L_{1} \cup L_{3}, L_{1}^{\prime} \cup L_{3}^{\prime}}\left(D_{1,3}\right)=D_{1,3}$. In both subcases that follow, our target will be to prove that $D_{1,3} \subseteq \sigma_{L_{1} \cup L_{2}, L_{1}^{\prime} \cup L_{2}^{\prime}}\left(D_{1,2}\right)$.

Subcase 3.a. If $\Delta \nsubseteq D_{1,2}$ then $\sigma_{L_{1} \cup L_{2}, L_{1}^{\prime} \cup L_{2}^{\prime}}\left(D_{1,2}\right)=D_{1,2} \cup \Delta \supseteq D_{1,2} \supseteq D_{1,3}$.

Subcase 3.b. If $\Delta \subseteq D_{1,2}$, we apply Lemma B.10 on $S$ and $S^{\prime}$ and let $D^{*}$ be a disc bounded by $L_{1} \cup L_{3}$ where $D^{*} \cap \Delta \subseteq\{x, y\}$. As $\left(L_{2}-\{x, y\}\right) \cap \Delta \neq \emptyset$, we imply that $L_{2}-\{x, y\} \subseteq \Sigma-D^{*}$. This means that $D^{*}=D_{1,3}$. We now have $D_{1,3}-\{x, y\} \subseteq$ $D_{1,3}-\left(D_{1,3} \cap \Delta\right)=D_{1,3}-\Delta \subseteq D_{1,2}-\Delta$. Therefore $\sigma_{L_{1} \cup L_{2}, L_{1}^{\prime} \cup L_{2}^{\prime}}\left(D_{1,2}\right)=\overline{D_{1,2}-\Delta} \supseteq$ $\overline{D_{1,3}-\{x, y\}}=D_{1,3}$.

\section{B.7 Proof of Theorem B.1}

Lemma B.14. Let $G$ be a triangulated $\Sigma$-plane graph without multiple edges and let ins be a uniform slope of order $k+1$ in $R_{G}$ for $k \geq 2$. Then, for any region $r$ of $R_{G}$, $\operatorname{ins}(\operatorname{bd}(r))=\bar{r}$. 
Proof. As ins is uniform we have that there exists a cycle $C^{\prime}$ of length $\leq 2 k$ such that $r \subseteq \operatorname{ins}\left(C^{\prime}\right)$. This means that $\mathbf{b d}(r) \subseteq \operatorname{ins}\left(C^{\prime}\right)$ and from axiom $[\mathbf{S 1}]$ we have that $\operatorname{ins}(\operatorname{bd}(r)) \subseteq \operatorname{ins}\left(C^{\prime}\right)$. Therefore, $\operatorname{ins}(\mathbf{b d}(r))=\bar{r}$.

We are now ready to prove the main technical result of this paper.

of Theorem B.1. Let ins be a uniform slope of order $k+1$ in $R_{G}$. We define the function big as follows. Let $N$ be a noose of $G$ with size $\leq k$. As $G$ is triangulated, Lemma B.7 implies that $N$ is the vibration of some of the cycles, say $C$ of $R_{G}$. Observe that $C$ has length $\leq 2 k$. In the trivial case $|N| \leq 1$ we define $\operatorname{big}(N)$ as the closed disk bounded by $N$ and containing all the vertices of $G$. For $|N| \geq 2$ we set $\operatorname{big}(N)=\sigma_{C, N}^{*}(\overline{\Sigma-\operatorname{ins}(C)})$.

We claim that the function big satisfies the majority axioms on $G$.

Proof of [M1]: Let $S=\left(L_{1}, L_{2}, L_{3}\right)$ be a $\Theta$-structure of size $\leq k$ where $L_{3} \subseteq \mathbf{b i g}\left(L_{1} \cup L_{2}\right)$. We will prove that $\operatorname{big}\left(L_{1} \cup L_{3}\right) \subseteq \operatorname{big}\left(L_{1} \cup L_{2}\right)$ or $\operatorname{big}\left(L_{2} \cup L_{3}\right) \subseteq \operatorname{big}\left(L_{1} \cup L_{2}\right)$. For this we distinguish two cases.

Special case. $S=\left(L_{1}, L_{2}, L_{3}\right)$ is trivial. Notice that $L_{i}, i=1,2,3$ have the same vertices, say $x, y$ of $G$ as endpoints. Also, from Lemma B.2, $e=\{x, y\}$ is an edge of $G$. We will first prove the following claim.

Claim. If $\left|L_{i} \cup L_{j}\right|=2,1 \leq i<j \leq 3$, then one, say $\Delta$, of the closed discs bounded by $L_{i} \cup L_{j}$ contains all the vertices of $G$ and $\operatorname{big}\left(L_{i} \cup L_{j}\right)=\Delta$.

Proof of Claim. The fact that $G$ is triangulated and without multiple edges implies that $G$ is 3-connected. Therefore, one of the closed discs, we denote it $\Delta$, bounded by $L_{i} \cup L_{j}$ contains all the vertices of $G$. It remains to prove that $\operatorname{big}\left(L_{1} \cup L_{2}\right)=\Delta$.

By Lemma B.7, the noose $L_{i} \cup L_{j}$, is a vibration of some cycle $C$ of $R_{G}$. As $\left|L_{i} \cup L_{j}\right|=2$, the only cycle of $R_{G}$ with this property is the boundary of $\mathbf{r}_{\{x, y\}}$. By Lemma B.14, $\operatorname{ins}(C)=\operatorname{ins}\left(\mathbf{b d}\left(\mathbf{r}_{\{x, y\}}\right)\right)=\overline{\mathbf{r}}_{\{x, y\}}$. From the definition of big we have that for all $i, j$, $1 \leq i<j \leq 3, \operatorname{big}\left(L_{i} \cup L_{j}\right)=\sigma_{C, L_{i} \cup L_{j}}^{*}\left(\overline{\Sigma-\overline{\mathbf{r}}_{\{x, y\}}}\right)$. Notice that $\overline{\Sigma-\overline{\mathbf{r}}_{\{x, y\}}} \cap V(G)=V(G)$ and Lemma B.11 yields that for $1 \leq i<j \leq 3, \sigma_{C, L_{i} \cup L_{j}}^{*}\left(\overline{\Sigma-\overline{\mathbf{r}}_{\{x, y\}}}\right) \cap V(G)=V(G)$, therefore $\mathbf{b i g}\left(L_{i} \cup L_{j}\right)$ should be equal to $\Delta$ and the claim holds.

We now distinguish the following subcases of the special case.

Subcase 1. $\left|L_{i}\right|=1, i=1,2,3$. Applying the claim above, we have that for $i, j, 1 \leq i<$ $j \leq 3, \operatorname{big}\left(L_{i} \cup L_{j}\right)$ is the closed disc bounded by $L_{i} \cup L_{j}$ and containing all the vertices of G. $L_{3} \subseteq \operatorname{big}\left(L_{1} \cup L_{2}\right)$ implies that either $L_{2}-\{x, y\} \subseteq \Sigma-\operatorname{big}\left(L_{1} \cup L_{3}\right)$ or $L_{1}-\{x, y\} \subseteq$ $\Sigma-\operatorname{big}\left(L_{2} \cup L_{3}\right)$. Then either $\mathbf{b i g}\left(L_{1} \cup L_{3}\right) \subseteq \operatorname{big}\left(L_{1} \cup L_{2}\right)$, or $\operatorname{big}\left(L_{2} \cup L_{3}\right) \subseteq \operatorname{big}\left(L_{1} \cup L_{2}\right)$.

Subcase 2. $\left|L_{i}\right|=1, i=1,2$ and $\left|L_{3}\right|=2$. From Lemma B.3 we have that $L_{1} \cup L_{3} \sim^{*}$ $L_{2} \cup L_{3}$. From the claim above, $\operatorname{big}\left(L_{1} \cup L_{2}\right)$ is the closed disc bounded by $L_{1} \cup L_{2}$ and containing all the vertices of $G$. Therefore, $\overline{\Sigma-\operatorname{big}\left(L_{1} \cup L_{2}\right)}=\operatorname{dif}\left(L_{1} \cup L_{3}, L_{2} \cup L_{3}\right)$. We 
now assume that $\mathbf{b i g}\left(L_{2} \cup L_{3}\right) \nsubseteq \mathbb{\mathbf { b i g }}\left(L_{1} \cup L_{2}\right)$. This can be rewritten as $\Sigma-\mathbf{b i g}\left(L_{1} \cup L_{2}\right) \nsubseteq$ $\Sigma-\operatorname{big}\left(L_{2} \cup L_{3}\right)$ which implies that $\operatorname{dif}\left(L_{1} \cup L_{3}, L_{2} \cup L_{3}\right) \nsubseteq \overline{\Sigma-\operatorname{big}\left(L_{2} \cup L_{3}\right)}$ and thus $\operatorname{dif}\left(L_{1} \cup L_{3}, L_{2} \cup L_{3}\right) \subseteq \operatorname{big}\left(L_{2} \cup L_{3}\right)$. We now have

$$
\begin{aligned}
\operatorname{big}\left(L_{1} \cup L_{3}\right) & =\sigma_{L_{2} \cup L_{3}, L_{1} \cup L_{3}\left(\mathbf{b i g}\left(L_{2} \cup L_{3}\right)\right)} \\
& =\overline{\operatorname{big}\left(L_{2} \cup L_{3}\right)-\operatorname{dif}\left(L_{1} \cup L_{3}, L_{2} \cup L_{3}\right)} \\
& \subseteq \overline{\Sigma-\operatorname{dif}\left(L_{1} \cup L_{3}, L_{2} \cup L_{3}\right)} \\
& =\operatorname{big}\left(L_{1} \cup L_{2}\right) .
\end{aligned}
$$

Subcase 3. $\left|L_{1}\right|=2$ and $\left|L_{i}\right|=1, i=2,3$. Observe that $L_{3} \subseteq \operatorname{big}\left(L_{1} \cup L_{2}\right)$ implies that $\operatorname{dif}\left(L_{1} \cup L_{2}, L_{1} \cup L_{3}\right) \subseteq \operatorname{big}\left(L_{1} \cup L_{2}\right)$. Therefore,

$$
\begin{aligned}
\operatorname{big}\left(L_{1} \cup L_{3}\right) & =\sigma_{L_{1} \cup L_{2}, L_{1} \cup L_{3}}\left(\mathbf{b i g}\left(L_{1} \cup L_{2}\right)\right) \\
& =\operatorname{big}\left(L_{1} \cup L_{2}\right)-\operatorname{dif}\left(L_{1} \cup L_{2}, L_{1} \cup L_{3}\right) \\
& \subseteq \operatorname{big}\left(L_{1} \cup L_{2}\right) .
\end{aligned}
$$

Subcase 4. $\left|L_{1}\right|=1$ and $\left|L_{2}\right|=2$ and $\left|L_{3}\right|=1$. This case is symmetric to Case 3 .

General Case. $S=\left(L_{1}, L_{2}, L_{3}\right)$ is non-trivial. Then, from Lemma B.9, there exist a nontrivial $\Theta$-structure $\left(P_{R}^{1}, P_{R}^{2}, P_{R}^{3}\right)$ of $G$ that is a vibration of $S$ where $P_{R}^{1}, P_{R}^{2}$ and $P_{R}^{3}$ are all paths of $R_{G}$. Lemma B.12 implies that $P_{3} \subseteq \mathbf{b i g}\left(P_{1} \cup P_{2}\right)$. As $\mathbf{b i g}\left(P_{1} \cup P_{2}\right)$ is a cycle of $R_{G}$, the definition of big implies that

$$
P_{3} \nsubseteq \nsubseteq \operatorname{ins}\left(P_{1} \cup P_{2}\right)
$$

Suppose now that $\mathbf{b i g}\left(P_{1} \cup P_{3}\right) \not \subset \mathbf{b i g}\left(P_{1} \cup P_{2}\right)$ and $\mathbf{b i g}\left(P_{2} \cup P_{3}\right) \not \subset \mathbf{b i g}\left(P_{1} \cup P_{2}\right)$ and we will show that this assumption leads to a contradiction. As $P_{i} \cup P_{j}, 1 \leq i<j \leq 3$, are cycles of $R_{G}$, the definition of big implies that

$$
\begin{array}{lll}
\operatorname{ins}\left(P_{1} \cup P_{2}\right) & \not \subset \operatorname{ins}\left(P_{2} \cup P_{3}\right) & \text { and } \\
\operatorname{ins}\left(P_{1} \cup P_{2}\right) & \not & \operatorname{ins}\left(P_{1} \cup P_{3}\right) .
\end{array}
$$

From (3) (4), and (5) we have that ins $\left(P_{1} \cup P_{2}\right) \cup \operatorname{ins}\left(P_{1} \cup P_{3}\right) \cup \operatorname{ins}\left(P_{2} \cup P_{3}\right)=\Sigma$ and this is a contradiction to [S2]. Therefore, we get that

$$
\operatorname{big}\left(P_{1} \cup P_{3}\right) \subseteq \operatorname{big}\left(P_{1} \cup P_{2}\right) \quad \text { or } \quad \operatorname{big}\left(P_{2} \cup P_{3}\right) \subseteq \operatorname{big}\left(P_{1} \cup P_{2}\right) .
$$

Applying now Lemma B.13 on each of the relations of (6), we conclude that either $\mathbf{b i g}\left(L_{1} \cup\right.$ $\left.L_{3}\right) \subseteq \operatorname{big}\left(L_{1} \cup L_{2}\right)$ or $\mathbf{b i g}\left(L_{2} \cup L_{3}\right) \subseteq \operatorname{big}\left(L_{1} \cup L_{2}\right)$.

Proof of [M2]: Let $N$ be a noose in $G$ where $|N|=2$ and $C$ be a path of $R_{G}$ where $N \sim^{*} C$ (in the case where $|N| \leq 1,[\mathbf{M} 2]$ follows from the bi-connectivity of $G$ ). By Lemma B.2, there exist an edge $e=\{x, y\}$ such that $(x, y)=\kappa_{G}(N)$. Clearly, if $r=\overline{\mathbf{r}}_{e}$ then $C=\mathbf{b d}(r)$. By Lemma B.14, ins $(C)=r$ and thus, $\overline{\Sigma-\operatorname{ins}(C)} \cap V(G)=V(G)$. By Lemma B.11, $\operatorname{big}(N) \cap V(G)=\sigma_{C, N}^{*}(\overline{\Sigma-\operatorname{ins}(C)}) \cap V(G)=V(G)$ and [M2] follows. 


\section{Departament de Llenguatges i Sistemes Informàtics Universitat Politècnica de Catalunya}

\section{Research Reports - 2003}

- LSI-03-1-R : Metaheuristics for the Edge-Weighted \$-Cardinality Tree Problem, Blesa, M.J. and Blum, C.

- LSI-03-2-R : Organizational and National Issues of an ERP Implementation in a Portuguese Company, Alvaro, J. and Esteves, J. and Pastor, J.A.

- LSI-03-3-R : A Complete Solid Model for Surface Rendering, Aguilera, A. and Ayala, D. and Rodríguez, J.

- LSI-03-4-R : Fast Neighborhood Operations for Images and Volume Datasets, Ayala, D. and Rodríguez, J.

- LSI-03-5-R : A Review of Integrity Constraint Maintenance and View Updating Techniques, Mayol, E. and Teniente, E.

- LSI-03-6-R : Porqpine: A Peer-to-Peer Search Engine, Bermúdez, J. and Pujol, J.M. and Sangüesa, R.

- LSI-03-7-R : KDSM Methodology for knowledge discovery from a domain where repeated very short serial measures with a blocking factor are present, Alvarado, G. and Cortés, U. and Gibert, K. and Rodas, J. and Rojo, J.E.

- LSI-03-8-R : On the Maximum Common Embedded Subtree Problem for Ordered Trees, Valiente, G.

- LSI-03-9-R : Searching by approximate personal-name matching, Camps, R. and Daudè, J.

- LSI-03-10-R : Two triangulations methods based on edge refinement, Ayala, D. and Pla, N. and Vigo, $\mathrm{M}$.

- LSI-03-11-R : Constrained Tree Inclusion, Valiente, G.

- LSI-03-12-R : Geometry of language and linguistic circuitry, Morrill, G.

- LSI-03-13-R : A simple yet useful approach to implementing UML Profiles in CASE tools ExtendedVersio , Cabot, J. and Gómez, C.

- LSI-03-14-R : Solving 'Still life' with Soft Constraints and Bucket Elimination, Larrosa, J. and Morancho, E.

- LSI-03-15-R : A fast hierarchical traversal strategy for multimodal visualization, Puig, A. and Tost, D.

- LSI-03-16-R : The complexity of deciding stability under FFS in the adversarial model, Àlvarez, C. and Blesa, M.J. and Díaz, J. and Fernández, A. and Serna, M.

- LSI-03-17-R : A Case Study on Building Conceptual Schemas by Refining General Ontologies, Conesa, J. and de Palol, X.

- LSI-03-18-R : Analysing similarity assessment in feature-vector case representations, Comas, J. and Cortés, U. and Núñez, H. and Poch, M. and Rodríguez-Roda, I. and Sànchez-Marrè, M.

- LSI-03-19-R : Análisis de medidas repetidas mediante el uso de la curva media como síntesis , 
Cortés, U. and Gibert, K. and Rodas, J. and Rojo, J.E.

- LSI-03-20-R : Statistical Strategies for Pruning All the Uninteresting Association Rules, Casas, G.

- LSI-03-21-R : Discovering Unbounded Episodes in Sequential Data, Casas, G.

- LSI-03-22-R : Query Containment with Negated IDB Predicates ExtendedVersion, Farré, C. and Teniente, E. and Urpí, T.

- LSI-03-24-R : Using concept lattices to mine functional dependencies, Baixeries, J.

- LSI-03-25-R : Adversarial models for prority-based networks, Àlvarez, C. and Blesa, M.J. and Fernández, A. and Serna, M.

- LSI-03-26-R : Characterization of Concept Lattices for Ordered Contexts, Casas, G.

- LSI-03-27-R : Computation of bisection width for random d-regular graphs, Serna, M. and Serras, J. and Wormald, N.C.

- LSI-03-28-R : Building and Using Quality Models for Complex Software Domains, Carvallo, J.P. and Franch, X. and Quer, C.

- LSI-03-29-R : Kernel Arquitecture for CAD/CAM in Shipbuilding Enviroments, González, J. and Gurrea, I. and Rodríguez, A. and Solano, Ll.

- LSI-03-30-R : Margin Maximization with Feed-forward Neural Networks: A Comparative Study with Support Vector Machines and AdaBoost, Carreras, X. and Màrquez, Ll. and Romero, E.

- LSI-03-31-R : Una notación algorítmica con genericidad y herencia junto con su relación con $\mathrm{C}++$ y Java, Mylonakis, N.

- LSI-03-32-R : Study on k-Shortest Paths with Behavioral Impedance Domain from the Intermodal Public Transportation System Perspective, Madriz-Lozada, E. and Pereira, H. and Perez, Ll.

- LSI-03-33-R : On the Complexity of Resolution with bounded Conjunctions, Borralleras, C. and Esteban, J.L. and Mossner, J.

- LSI-03-34-R : Generic Algorithms for the Generation of Combinatorial Objects, Martínez, C. and Molinero, $\mathrm{X}$.

- LSI-03-35-R : An Efficient Generic Algorithm for the Generation of Unlabelled Cycles, Martínez, C. and Molinero, X.

- LSI-03-36-R : CBR and MBR techniques: review for an application in the emergencies domain, Mérida-Campos, C. and Rollón, E.

- LSI-03-37-R : Using NLP tools in the Specification Phase, Castell, N. and Hernández, A.

- LSI-03-38-R : Classificació automàtica amb KLASS de les dades de procés d'una EDAR, Flores, $\mathrm{X}$. and Gibert, K. and Rodríguez-Roda, I.

- LSI-03-39-R : VolumeEVM: A new surface/volume integrated model, Ayala, D. and Rodríguez, $\mathrm{J}$.

- LSI-03-40-R : MKtrees: Construction and Applications, Brunet, P. and Franquesa, M.

- LSI-03-41-R : From Supply Chains to Demand NetworksAgents in Retailing: The Electrical Bazaar , Almirall, E. and Brito, I. and Cortés, U. and Silisque, A. 
- LSI-03-42-R : Fixed-Parameter Algorithms for the k, r-Center in Planar Graphs and Map Graphs , Demaine, E.D. and Fomin, F.V. and Hajiaghayi, M. and Thilikos, D.M.

- LSI-03-43-R : Dominating sets and local treewidth, Fomin, F.V. and Thilikos, D.M.

- LSI-03-44-R : Res2 Lower Bounds for Tseitin Formulas, Bonet, M. and Esteban, J.L.

- LSI-03-45-R : Collision Queries: Models and Algorithms, Brunet, P. and Franquesa, M.

- LSI-03-46-R : Collision Prediction Using MKtrees: Broad Phase and Refinement Levels of the Narrow Phase, Brunet, P. and Franquesa, M.

- LSI-03-48-R : Bidimensional Parameters and Local Treewidth, Demaine, E.D. and Fomin, F.V. and Hajiaghayi, M. and Thilikos, D.M.

- LSI-03-50-R : A distributed algorithm to find Hamiltonian cycles in Gnp random graphs, Levy, J. and Louchard, G. and Petit, J.

- LSI-03-51-R : A characterization of universal stability in the adversarial queuing model, Àlvarez, C. and Blesa, M.J. and Serna, M.

- LSI-03-52-R : Automatic Generation of Polynomial Loop Invariants for Imperative Programs, Kapur, D. and Rodríguez, E.

- LSI-03-53-R : Speeding up rendering of hybrid surface and volume models, Ferré, M. and Puig, A. and Tost, D.

- LSI-03-54-R : A Simple and Fast Approach for Solving Problems on Planar Graphs, Fomin, F.V. and Thilikos, D.M.

Hardcopies of reports can be ordered from:

Núria Sanchez

Departament de Llenguatges i Sistemes Informàtics

Universitat Politècnica de Catalunya

Campus Nord, Mòdul C6

Jordi Girona Salgado, 1-3

03034 Barcelona,Spain

nurias@1si.upc.es

See also the Departament WWW pages, http://www.lsi.upc.es/ 